\title{
GQDs-MSNs nanocomposite nanoparticles for simultaneous intracellular drug delivery and fluorescent imaging
}

\author{
Dorota Flak • Lucja Przysiecka • \\ Grzegorz Nowaczyk • Blażej Scheibe • \\ Mikołaj Kościński • Teofil Jesionowski • Stefan Jurga
}

Received: 13 April 2018 / Accepted: 2 November 2018/Published online: 17 November 2018

(C) The Author(s) 2018

\begin{abstract}
Although number of stimuli-responsive drug delivery systems based on mesoporous silica nanoparti-
\end{abstract}

Electronic supplementary material The online version of this article (https://doi.org/10.1007/s11051-018-4416-y) contains supplementary material, which is available to authorized users.

D. Flak $(\bowtie) \cdot$ Ł. Przysiecka $\cdot$ G. Nowaczyk $\cdot$ B. Scheibe • M. Kościński $\cdot$ S. Jurga

NanoBioMedical Centre, Adam Mickiewicz University in Poznań, Umultowska 85, 61-614 Poznań, Poland e-mail:dorfla@amu.edu.pl

\author{
Ł. Przysiecka \\ e-mail: lucprz@amu.edu.pl \\ G. Nowaczyk \\ e-mail: nowag@amu.edu.pl \\ B. Scheibe \\ e-mail: blasch@amu.edu.pl \\ M. Kościński \\ e-mail: mikolaj.koscinski@amu.edu.pl \\ S. Jurga \\ e-mail: stjurga@amu.edu.pl \\ M. Kościński \\ Department of Physics and Biophysics, Poznań University of Life \\ Sciences, Wojska Polskiego 38/42, 60-637 Poznań, Poland \\ T. Jesionowski \\ Institute of Chemical Technology and Engineering, Faculty of \\ Chemical Technology, Poznan University of Technology, \\ Berdychowo 4, 60-965 Poznań, Poland \\ e-mail: Teofil.Jesionowski@put.poznan.pl
}

cles (MSNs) have been developed, the simultaneous real-time monitoring of carrier in order to guarantee proper drug targeting still remains as a challenge. GQDs-MSNs nanocomposite nanoparticles composed of graphene quantum dots (GQDs) and MSNs are proposed as efficient doxorubicin delivery and fluorescent imaging agent, allowing to monitor intracellular localization of a carrier and drug diffusion route from the carrier.

Graphene quantum dots (average diameter $3.65 \pm$ $0.81 \mathrm{~nm}$ ) as a fluorescent agent were chemically immobilized onto mesoporous silica nanoparticles (average diameter $44.08 \pm 7.18 \mathrm{~nm}$ ) and loaded with doxorubicin. The structure, morphology, chemical composition, and optical properties as well as drug release behavior of doxorubicin (DOX)-loaded GQDs-MSNs were investigated. Then, the in vitro cytotoxicity, cellular uptake, and intracellular localization studies were carried out. Prepared GQDs-MSNs form stable suspensions exhibiting excitation-dependent photoluminescence (PL) behavior. These nanocomposite nanoparticles can be easily DOX-loaded and show $\mathrm{pH}$ - and temperaturedependent release behavior. Cytotoxicity studies proved that GQDs-MSNs nanocomposite nanoparticles are nontoxic; however, when loaded with drug, they enable the therapeutic activity of DOX via its active delivery and release. GQDs-MSNs owing to their fluorescent properties and efficient in vitro cellular internalization via caveolae/lipid raft-dependent endocytosis show a high potential for the optical imaging, including the simultaneous real-time optical tracking of the loaded drug during its delivery and release. 
Keywords Graphene quantum dots · Mesoporous silica nanoparticles $\cdot$ Nanocarriers $\cdot$ Cellular uptake $\cdot$ Drug delivery $\cdot$ Real-time monitoring of drug release . Bioimaging · Nanomedicine

\section{Introduction}

The development of theranostic nanoplatforms for simultaneous diagnosis and therapy, particularly in cancer treatment, is enabled by the combination of nanomaterials with different functionalities. Among a variety of multifunctional nanoplatforms, mesoporous silica-based nanostructures and nanocomposite materials has been recognized and widely studied as promising drug carriers owing to their mesoporous structure, their unique properties, such as large surface area and pore volume, high chemical stability, reactive surface, but also cell membrane-penetration ability and low cytotoxicity (Lee et al. 2011). Mesoporous silica nanoparticles (MSNs) have been employed so far as an efficient carrier of various therapeutics, but also imaging agents, including other functional nanostructured materials. It was proposed that MSNs-based drug delivery systems facilitate controlled delivery and release of anticancer drugs, thus enhancing their therapeutic efficiency along with diminishing their side effects in comparison to standard drug administration (Bharti et al. 2015). Up to date, many MSNs-based theranostic nanoplatforms for bioimaging, drug delivery, and therapy have been developed, taking the advantage of different nanoparticles as capping agents, such as, e.g., $\mathrm{Fe}_{3} \mathrm{O}_{4}$ (Lee et al. 2010), Au (Ma et al. 2012), CdS (Lai et al. 2003), or embedded into MSNs as a core, e.g., $\mathrm{Fe}_{3} \mathrm{O}_{4}$ (Yao et al. $2017 \mathrm{a}, \mathrm{b})$, but also paramagnetic ions $\left(\mathrm{Gd}^{3+}, \mathrm{Mn}^{2+}\right)$ incorporation ( $\mathrm{Lin}$ et al. 2004) or their chelates (Cao et al. 2015). Another approach also includes the advantage of different release stimuli, such as $\mathrm{pH}$, redox potential, adenosine triphosphate gradient, enzymes, temperature, and multi-stimuli-responsive systems (Moreira et al. 2016).

Number of developed stimuli-responsive drug delivery systems based on MSNs have been developed so far; however, the simultaneous real-time monitoring of the drug carrier in order to guarantee proper drug targeting remains as a challenge. Despite the great potential of MSNs as an efficient drug carrier, these nanoparticles cannot itself emit a fluorescence signal allowing for their detection, however not only when the drug has been released, but also before the release, allowing to monitor intracellular localization of a carrier and diffusion route of the drug from the carrier. This issue has been addressed by the fluorescent labeling of MSNs by capping or encapsulation with fluorescence organic dyes, e.g., fluorescein isothiocyanite ( $\mathrm{Lu}$ et al. 2009) upconversion nanoparticles (Niu et al. 2014) and more recently with quantum dots (QDs) (Zhang et al. 2016; Yao et al. 2017a).

QDs are fluorescent nanocrystals, which are considered as an ideal fluorescent agent for bioimaging owing to their many superior properties compared to organic dyes (Resch-Genger et al. 2008). These properties are photostability, broad excitation wavelength, narrow emission, continuous and broad absorption spectra, and finally susceptibility to surface functionalization, including biomolecules. The most commonly used so far QDs contain heavy metals (e.g., CdHgTe, CdTeSe@CdZnS, CdSe@ZnS), which cause undesirable biological and environmental effects, and thus limit their use in biological applications. Graphene quantum dots (GQDs) have emerged as an alternative and a new class of QDs with fluorescent properties (Wen et al. 2015). GQDs are kind of zero-dimensional small graphene sheets fragments, in which the electronic transport is confined in three spatial dimensions. Due to the quantum confinement and edge effects, GQDs possess a non-zero band-gap, and therefore emit luminescence upon the excitation. GQDs are built-up from carbon, which is abundant in the biological systems; therefore, they are considered as a biocompatible nontoxic material. Moreover, GQDs show a molecule-like character and contain a number of carboxylic, epoxy and hydroxyl groups; therefore, they can be easily dissolved in water-based solvents and are easy for further functionalization. Finally, GQDs exhibit stable photoluminescence and superior resistance to photobleaching in comparison to traditional semiconductor QDs and organic dyes. Yao et al. (Yao et al. $2017 \mathrm{a}, \mathrm{b})$ reported on the GQDs as caps and local photothermal generators and magnetic mesoporous silica nanoparticles as drug carriers and magnetic thermoseeds, which exhibited strong synergetic effect, resulting in high efficiency to kill cancer cells. Later, Yao et al. (Yao et al. 2017b) reported on GQDs-capped MSNs with a potential for combined chemo- and photothermal cancer therapy, showing $\mathrm{pH}$ - and temperature-responsive doxorubicin release, and NIRinduced photothermal cytotoxicity. A similar system has 
been reported by Huang et al. (Huang et al. 2016), where GQDs-decorated MSNs have been prepared through the electrostatic interaction in contrast to previously mentioned studies, and have been successfully used for aspirin loading and its release. The imaging capacity of GQDs/MSNs system next to the drug delivery has been reported for the first time by Chen et at. (Chen et al. 2014), where GQDs were capped onto MSNs through an acid-cleavable acetal bond, and hence the acidic $\mathrm{pH}-$ triggered doxorubicin release from mesopores.

Summarizing, taking the advantage of both components properties, the combination of MSNs and GQDs may provide a new strategy for integrated nanocomposite system for an efficient optical bioimaging and drug delivery system, which is yet to be developed.

In order to address current challenges concerning the theranostic nanoplatform, such as increase of the efficient uptake and accumulation in cancer cells, effective drug delivery, and controlled release with simultaneous imaging and real-time monitoring capability, GQDsMSNs nanocomposite nanoparticles were prepared by the immobilization of GQDs onto MSNs, which were then loaded with doxorubicin as a model anticancer drug. The release studies were performed in $\mathrm{pH}-$ and temperature-dependent manner. So far, most of the GQDs/MSNs-integrated nanoplatforms have been studied mainly for controlled drug delivery, and also for other therapeutic modalities, but their potential as bioimaging agent has been rather underestimated and dominated by therapeutic activities of the system. Therefore, further in this manuscript, the focus is on the detailed investigation on the cellular uptake and intracellular translocation of the GQDs-MSNs, which are the basis for the possibility of the simultaneous imaging and real-time monitoring not only of the drug but also the drug carrier, which has not been well presented until now. The efficiency of prepared GQDsMSNs nanocomposite nanoparticles for the simultaneous drug delivery and release next to the bioimaging was evaluated using HeLa cancer cells as a model cellular system.

\section{Experimental}

Materials and methods

Sodium citrate tribasic dehydrate (NaCitr, $\geq 99 \%$ ), thiourea (TU, $\geq 99 \%$ ), tetraethyl orthosilicate (TEOS, 98\%), hexadecyltrimethylammonium bromide $(\mathrm{CTAB}, \geq$ $99 \%), \quad N-(3-d i m e t h y l a m i n o p r o p y l)-N^{\prime}-$ ethylcarbodiimide hydrochloride (EDC), (3aminopropyl) triethoxysilane (APTES, $\geq 98 \%$ ), and phosphate-buffered saline (PBS) were purchased from Sigma-Aldrich. Ethanol (EtOH, 99.8\%), methanol (MetOH, 99.8\%), and hydrochloric acid $(\mathrm{HCl}, 35-$ $38 \%$ ) were purchased from POCH Basic. Dulbecco's Modified Eagle's medium (DMEM), Hanks Balanced Salt solution, fetal bovine serum (FBS), Trypsine-EDTA $(0.25 \%)$, penicillin-streptomycin, glutaraldehyde solution, and agarose were purchased from Sigma-Aldrich and used as received. WST-1 Cell Proliferation Assay Kit was purchased from Clontech. LIVE/DEAD® Viability/Cytotoxicity Kit for mammalian cells was obtained from Thermo Fisher Scientific. Formaldehyde methanol free, osmium tetroxide solution, uranyl acetate, and Embed-It ${ }^{\mathrm{TM}}$ Low Viscosity Epoxy Kit were purchased from Polysciences.

\section{Preparation of GQDs}

GQDs were prepared via the hydrothermal method (Permatasari et al. 2016; Qu et al. 2014), with the minor modification, using NaCitr (instead of citric acid) as a carbon precursor and TU as a base. NaCitr and TU reagents were mixed in the molar ratio of 1:3 (1.3 g:1.15 g), respectively. The reaction was run in water-based $(25 \mathrm{ml})$ solution of both reagents in Teflon-lined stainless autoclave at $180{ }^{\circ} \mathrm{C}$ for $8 \mathrm{~h}$. The formation of GQDs was indicated by the color change of the reaction solution to the orange one. Obtained postreaction solution was then given to vacuum drying at $60{ }^{\circ} \mathrm{C}$ and pressure of 200 mbar in order to remove the excess water. The final product was precipitated by adding EtOH to the solution and collected by centrifugation (13.2 rpm, $15 \mathrm{~min}$ ), and dried at $60{ }^{\circ} \mathrm{C}$. The obtained solid material can be easily dispersed in water.

\section{Preparation of MSNs}

MSNs, as a carrier for GQDs, were synthesized using the previously reported template-directed sol-gel method, with the minor modifications (Nooney et al. 2002). In a typical used synthesis procedure $0.5 \mathrm{~g} \mathrm{CTAB}$, as the structure-directing agent, was dispersed in $240 \mathrm{ml}$ of deionized water $\left(\mathrm{H}_{2} \mathrm{O}_{\mathrm{d}}\right)$ in a closed vessel under vigorous stirring at room temperature. After the solution 
became homogenous, its $\mathrm{pH}$ was adjusted to 11 with the $1 \mathrm{M}$ (molar) $\mathrm{NaOH}$ solution and heated up to $80^{\circ} \mathrm{C}$. Subsequently, $2.5 \mathrm{ml}$ of TEOS as the silicon source was dropwise added and the reaction solution was further stirred continuously for $3 \mathrm{~h}$, until white precipitated was obtained. As-obtained product was collected by centrifugation $(24,000 \mathrm{rpm}, 15 \mathrm{~min})$ and washing with $\mathrm{MetOH}$ and then dried overnight at $60^{\circ} \mathrm{C}$. In order to remove $\mathrm{CTAB}$ template, the final dried product was refluxed for $24 \mathrm{~h}$ in a mixture of $160 \mathrm{ml} \mathrm{MetOH}$ and $9 \mathrm{ml} \mathrm{HCl}(\sim 37 \%)$. The obtained MSNs were centrifuged and washed with $\mathrm{MetOH}$ and $\mathrm{H}_{2} \mathrm{O}_{\mathrm{d}}$, and finally dried at $60{ }^{\circ} \mathrm{C}$.

Preparation GQDs-MSNs nanocomposite nanoparticles

Prior to the GQDs-MSNs nanocomposites preparation, as-prepared MSNs were firstly amino-functionalized. For this purpose, $0.04 \mathrm{~g}$ MSNs was dispersed in EtOH and then treated with $0.8 \mathrm{ml}$ APTES under the stirring. After $24 \mathrm{~h}$, the suspension was centrifuged (13.2 rpm, $15 \mathrm{~min}$ ) and washed with $\mathrm{EtOH}$ repeatedly for three times, in order to remove unreacted APTES. The prepared GQDs were then covalently immobilized onto amino-functionalized MSNs ( $\mathrm{NH}_{2}$-MSNs) with the use of carbodiimide crosslinker chemistry. The aminofunctionalized MSNs were dispersed in PBS-based solution $(\mathrm{pH}=5.8)$ of GQDs $(1 \mathrm{mg} / \mathrm{ml})$ and added with $0.2 \mathrm{ml}$ of EDC linker solution $(20 \mathrm{mg} / \mathrm{ml})$. After $24 \mathrm{~h}$, the unbound QDs were removed by successive centrifugation and washing with $\mathrm{EtOH}$ and $\mathrm{H}_{2} \mathrm{O}_{\mathrm{d}}$.

\section{DOX-loading and release studies}

Doxorubicin $\mathrm{HCl}$ (DOX, Carbosynth Limited), a model chemotherapy drug, was non-covalently loaded onto prepared GQDs-MSNs nanocomposite nanoparticles, as well as onto un-modified MSNs. Briefly, suspension of nanocarrier was mixed with DOX solution (both prepared in PBS $\mathrm{pH}=7.4$, in a weight ratio of $2: 1$, respectively). A resulting reaction solution was given to continuous stirring for $24 \mathrm{~h}$ at the room temperature and in the dark. An obtained red-purple precipitate was separated by centrifugation, washed with PBS several times, and dried in air at $60^{\circ} \mathrm{C}$. The efficiency of DOX loading was determined by finding the difference in the DOX concentration in the solution before and after loading. DOX concentration was measured spectrophotometrically at $485 \mathrm{~nm}$. Percentage of the loaded drug was calculated according to the following equation:

$\%$ DOX $_{\text {loaded }}=\left[\left(\mathrm{C}_{\text {DOX } 0}-\mathrm{C}_{\text {DOX F }}\right) / \mathrm{C}_{\text {DOX } 0}\right] * 100$

where $C_{\mathrm{DOX} 0}$ and $C_{\mathrm{DOX} F}$ are the initial and final DOX concentration in the reaction mixture.

To investigate drug release behavior from prepared GQDs-MSNs nanocomposites, as well as MSNs, $0.5 \mathrm{mg}$ DOX-loaded nanocarrier was dispersed in $2 \mathrm{ml}$ of PBS with $\mathrm{pH} 4.5,5.0,6.5$, and 7.4 and given to continuous shaking under dark conditions. The temperature of PBS solution was kept constant 37 or $50{ }^{\circ} \mathrm{C}$. After predetermined time intervals, the samples were centrifuged, then supernatant samples were withdrawn and replaced with fresh PBS for continuous drug release. The amount of released DOX was determined spectrophotometrically at $485 \mathrm{~nm}$.

Physicochemical characterization

The morphology and size of prepared GQDs were investigated using the transmission electron microscopy (TEM) and atomic force microscopy (AFM). TEM images were recorded with a high-resolution and analytical HRTEM Jeol ARM 200F operating at $200 \mathrm{kV}$ or TEM Jeol JEM-1400 operating at $120 \mathrm{kV}$. AFM images were recorded in PeakForce Tapping® mode with the Icon Bruker microscope. Silicon nitride ScanAsyst-Air probes were used during the experiment and a freshly cleaved mica as a substrate. The crystal structure was characterized by X-ray diffraction (XRD) using Empyrean (PANalytical) diffractometer with $\mathrm{Cu} \mathrm{K} \alpha$-filtered radiation $(1.54 \AA)$. The vibrational properties of prepared samples were analyzed via attenuated total reflection (ATR) technique with a Tensor 27 spectrometer (Bruker), equipped with a MCT detector and a horizontal triple reflection PIKE MIRacle ${ }^{\text {TM }}$ ATR accessory. Raman spectra were collected using an in Via Renishaw Raman Microscopy system with a 633-nm He/Ne laser and $1800 \mathrm{~g} / \mathrm{mm}$ grating. The laser light was focused on the sample with a $\times 50 / 0.75$ microscope objective (LEICA). $\mathrm{N}_{2}$ adsorption-desorption isotherms were obtained on Sorptometr Quantachrome NOVA 1000e. The surface area (SSA) and pore volume have been determined based on low temperature $\mathrm{N}_{2}$ sorption and estimated applying Brunauer-Emmett-Teller (BET) method and Barrett-Joyner-Halenda algorithm. Zeta potential and particle size distribution of prepared GQDs were measured on Zetasizer Nano ZS (Malvern Instruments 
Ltd.) based on electrophoretic light scattering (ELS) and non-invasive light scattering method (NIBS), respectively. The chemical composition of prepared GQDs was investigated using X-ray photoelectron spectroscopy (XPS). XPS spectra were obtained with an Sphera II photoelectron energy analyzer (Scienta Omicron) with the monochromatized Al K $\alpha$ X-ray source mounted inside the UHV system. The absorbance and fluorescence excitation and emission spectra were acquired on UV-Vis-NIR spectrophotometer (Perkin Elmer lambda 950) and spectrofluorometer (FluoroSENS Gilden Photonics). The fluorescent quantum yield (QY) of prepared GQDs solutions in water was determined by applying the following equation:

$\mathrm{QY}_{\text {sample }}=\mathrm{QY}_{\mathrm{st}}\left(\frac{I_{\text {sample }}}{I_{\mathrm{st}}}\right)\left(\frac{n_{\text {sample }}^{2}}{n_{\mathrm{st}}^{2}}\right)\left(\frac{A_{\text {st }}}{A_{\text {sample }}}\right)$

where QY is the quantum yield, $I$ is the measured integrated emission, $A$ is the absorbance at the excitation wavelength, and $n$ is refractive index of the solvent. As a standard, the quinine sulphate solution in $0.1 \mathrm{M}$ sulfuric acid solution was used $(\mathrm{QY}=54.6 \%)$.

Biochemical characterization

\section{Cell culture}

Human cervical cancer cell line HeLa obtained from American Type Culture Collection (ATCC) and human fibroblast cell line MSU1.1 obtained from Prof. C. Kieda (CBM, CNRS, Orléans, France) were used for in vitro studies. Cells were cultured in a complete medium DMEM supplemented with 10\% FBS, $100 \mathrm{units} / \mathrm{ml}$ penicillin, and $100 \mu \mathrm{g} / \mathrm{ml}$ streptomycin, and grown at $37^{\circ} \mathrm{C}$ in humidified atmosphere containing $5 \% \mathrm{CO}_{2}$.

\section{In vitro cytotoxicity assays}

In order to determinate the cytotoxicity of GQDs, MSNs, and GQDs-MSNs nanocomposite nanoparticles, as well as DOX-loaded nanocomposite nanoparticles, HeLa and MSU1.1 cells were treated with increasing concentration of nanoparticles (from 20 to $400 \mu \mathrm{g} / \mathrm{ml}$ ) and incubated for $24 \mathrm{~h}$ at $37{ }^{\circ} \mathrm{C}$ under a $5 \% \mathrm{CO}_{2}$ atmosphere. Cells without any treatment were used as a negative control. The effect of the GQDs on cell viability was determined by WST-1 assay according to manufacturer's instructions. Briefly, $10 \mu \mathrm{l}$ of WST-1 solution was added to each well of 96-well plate and further incubated. After $2 \mathrm{~h}$, the absorbance was measured with a microplate reader (Anthos Zenyth 340rt) at 450 versus a $650 \mathrm{~nm}$ reference. The relative cell viability (\%) was expressed as a percentage relative to the negative control. Data are reported as the average \pm standard deviation (SD) of experiments performed in triplicate. The cytotoxicity analysis was also performed for chemical inhibitors of cellular uptake (see, Fig. S4 in ESM).

The effect of the nanoparticles on cell viability was also determined using Live/Dead assay kit and analyzed by InCell Analyzer apparatus (GE Healthcare). Briefly, cultured adherent cells in a 96-well plate, previously coincubated for $24 \mathrm{~h}$ with nanoparticles solutions at increasing concentration mentioned before, were prepared. Cells non-treated and treated with $50 \mathrm{v} / \mathrm{v} \%$ dimethyl sulfoxide were used as negative and positive control (DMSO), respectively. Cells were washed with PBS, then a Live/Dead Viability Kit (Life Technologies) composed of two fluorescent dyes, calcein-AM and ethidium homodimer (EthD-1), for the staining of live and dead cells were used. Thus, in live cells, green fluorescence derived from calcein was observed in cytoplasm, whereas EthD-1 enters dead cells and was observed as red fluorescence in nucleus. Three repetitions for each condition were carried out. The images were acquired from 20 fields from each well, and then statistically analyzed by InCell Developer Toolbox software. The total cell number was normalized to the nontreated control group. Data are expressed as the average \pm standard deviation (SD) of three different experiments.

Cell uptake of GQDs- and GQDs-MSNs-imaging studies

\section{CLSM imaging}

To investigate the GQDs and GQDs-MSNs nanocomposites' ability to cell penetration and their intracellular distribution, as well as to monitor DOX delivery and release, confocal microscopy was performed on cancer cell line (HeLa). Briefly, cells were plated on a chambered Lab-Tek dish $\left(1 \times 10^{4}\right.$ cells/well $)$, grown overnight, and then were incubated at $37{ }^{\circ} \mathrm{C}$ with GQDs, GQDs-MSNs nanocomposites $(20 \mu \mathrm{g} / \mathrm{ml})$ for 3 or $24 \mathrm{~h}$. The cells were then rinsed three times with PBS 
(pH 7.4). The distribution of GQDs, GQDs-MSNs nanocomposites and doxorubicin was analyzed using a confocal laser scanning microscope (CLSM, FV1000, Olympus). Colocalization of DOX with GDQ-MSN was analyzed via Pearson's coefficient constant.

\section{Tracking pathway of cellular uptake}

In order to block energy-dependent mechanisms of GQDs-MSNs uptake, the grown HeLa cells were incubated at $4{ }^{\circ} \mathrm{C}$ for $1 \mathrm{~h}$. Media was then replaced with cold serum-free DMEM containing $20 \mu \mathrm{g} / \mathrm{ml}$ of GQDsMSNs and incubated for another $3 \mathrm{~h}$ at $4{ }^{\circ} \mathrm{C}$. Afterward, cells were rinsed with PBS, maintained in phenol redfree medium, and imaged using a laser scanning confocal microscope (CLSM, Olympus FV1000).

The influence of different endocytic inhibitors on the cellular uptake of GQDs-MSNs was also assessed. Briefly, the seeded HeLa cells were incubated separately with (1) methyl- $\beta$-cyclodextrin $(2,5 \mathrm{mg} / \mathrm{ml})$, as an inhibitor of caveolae/lipid raft-dependent endocytosis; (2) chlorpromazine hydrochloride $(5 \mu \mathrm{g} / \mathrm{mL})$, as an inhibitor of clathrin-mediated endocytosis; and (3) wortmannin $(150 \mathrm{ng} / \mathrm{mL})$, as macropinocytosis inhibitor, for $1 \mathrm{~h}$ at $37^{\circ} \mathrm{C}$. Subsequently, cells were incubated with $20 \mu \mathrm{g} / \mathrm{ml}$ of GQDs-MSNs nanoparticles nanocomposite for $3 \mathrm{~h}$ and imaged as mentioned above. The concentration of different inhibitors were chosen based on performed earlier their cytotoxicity analysis (Fig. S2 in ESM).

\section{TEM imaging}

The cellular uptake and distribution of GQDs-MSNs nanocomposites in cells was further analyzed by TEM (Jeol JEM-1400) according to Graham and Orenstein (Graham and Orenstein 2007) procedure with minor modification. Briefly, HeLa cells previously incubated with $100 \mu \mathrm{g} / \mathrm{ml}$ of GQDs or GQDs/MSNs nanocomposites for $4 \mathrm{~h}$ were fixed with $2.5 \%$ glutaraldehyde solution for $2 \mathrm{~h}$ and then postfixed in $1 \%$ osmium tetroxide for $1 \mathrm{~h}$ at room temperature. Then, samples were dehydrated through a graded series of ethanol concentrations $(50,70,80,90,96$, and 100\%) and embedded in epoxy resin. Ultrathin sections were prepared using ultramicrotome (RMC PowerTome PT-XL), collected onto TEM grids, stained with $1 \%$ uranyl acetate, and imaged under Jeol JEM-1400 TEM.

\section{Results and discussion}

Preparation and characterization of GQDs and GQDs-MSNs

For the synthesis of GQDs, the bottom-up approach was chosen, as it is reported to yield better quality GQDs concerning their morphology, size distribution, and optical properties (Bacon et al. 2014). After the hydrothermal reaction, a bluish solution with blue emission under UV light was obtained. From the HRTEM images (Fig. S1a,b in ESM), a clear interplanar distance of about $0.35 \mathrm{~nm}$ can be found as marked particularly in the Fig. S1b, which corresponds to that of the (002) d-spacing of graphite (Qu et al. 2013) confirming that prepared GQDs have a graphite-like nature. As presented in the HRTEM images, the crystal structure of GQDs is strongly defected; hence, it is difficult to indicate accurate hexagonal ordering typical for graphene. Prepared GQDs are uniform in size (ranging from 1.5 to $7.6 \mathrm{~nm}$ ), with an average particles diameter of $3.65 \mathrm{~nm}$ (Fig. S1c in ESM). AFM images (Fig. S1 e, f in ESM) confirm that uniform GQDs were prepared, as they show a topographic height of nanostructures up to $1.15 \mathrm{~nm}$. Raman spectrum of prepared GQDs reveals prominent D, G, D', and 2D bands at $1328.6 \mathrm{~cm}^{-1}, 1575.8 \mathrm{~cm}^{-1}, 1611.2 \mathrm{~cm}^{-1}$, and $2642.6 \mathrm{~cm}^{-1}$, respectively. $\mathrm{G}$ band is a result of in-plane vibrations of $\mathrm{sp}^{2}$ bonded carbon atoms, whereas $\mathrm{D}$ and D' bands are due to the out of plane vibrations attributed to the presence of structural defects. Found $I_{D} / I_{G}$ ratio is around 0.86 , which indicates that $\mathrm{sp}^{2}$ bonds of the carbon are disrupted by the presence of oxygen-containing functional groups (originating from the precursor), and therefore prepared GQDs are considered as highly defected materials.

The XPS elemental analysis of prepared GQDs reveals the presence of carbon, oxygen, nitrogen, sulfur, and sodium. The survey and relevant core level C $1 \mathrm{~s}, \mathrm{O}$ $1, \mathrm{~N} 1 \mathrm{~s}$ spectra and $\mathrm{S} 2 \mathrm{~s}, \mathrm{~S} 2 \mathrm{p}$ region spectra are given in Fig. S2 in ESM. C 1s core level spectrum is composed of three peaks at around $284.5 \mathrm{eV}, 286.0 \mathrm{eV}$, and $288.2 \mathrm{eV}$ that can be assigned to the $\mathrm{sp}^{2} \mathrm{C}$ in graphene (C-C, $\mathrm{C}=\mathrm{C}$ bonds), $\mathrm{sp}^{3} \mathrm{C}$ in $\mathrm{C}-\mathrm{O}, \mathrm{C}-\mathrm{N}$ bonds and $\mathrm{O}-$ $\mathrm{C}=\mathrm{O}$, respectively ( $\mathrm{Qu}$ et al. 2013). The O 1s core level peaks at around 531.3 and $532.6 \mathrm{eV}$ correspond to oxygen in states of $\mathrm{C}=\mathrm{O}, \mathrm{C}-\mathrm{O}-\mathrm{C} / \mathrm{C}-\mathrm{OH}$, respectively ( $\mathrm{Qu}$ et al. 2013). As shown, $\mathrm{C}=\mathrm{O}$-related oxygen states are dominating. The $\mathrm{C}=\mathrm{O}$ groups are formed by the intramolecular dehydrogenation of carbon precursor, 
and the other $\mathrm{C}-\mathrm{O}-\mathrm{C} / \mathrm{C}-\mathrm{OH}$ are remaining surface and edge-related epoxy groups and carboxylic functional groups, resulting from the incomplete dehydrogenation and carbonization during the synthesis. Moreover, N 1s core level spanning from around 396.0 to $403.0 \mathrm{eV}$ is clearly resolved, as well as S 2 s and S 2 p core levels at around 227.0 and $163.0 \mathrm{eV}$, respectively (Qu et al. 2013). Elemental XPS analysis reveals that prepared GQDs contain 72.34 at\% of C, which is a high content in comparison to other results (Hao et al. 2015), 13.97 at $\%$ of O, 4.97 at $\%$ of $\mathrm{N}$ and 5.39 at $\%$ of S, hence confirming $\mathrm{N}$ and S-doping of GQDs.

These results confirm that obtained GQDs are rich in carbon, doped with $\mathrm{N}$ and $\mathrm{S}$ atoms, but still contain number of oxygen-containing functional groups and exhibit disordered and disrupted structure, what is likely for zero-dimensional (0D) nanostructures in comparison to two-dimensional (2-D) graphene nanosheets (Kozak et al. 2016).

During the synthesis of MSNs, the $\mathrm{pH}$ and temperature were carefully controlled in order to obtain nanostructures with size below $50 \mathrm{~nm}$, as their eventual biomedical application is foreseen. As shown in the TEM images (Fig. 1a), obtained silica nanoparticles are spherical and dendritic structures, with the average particle size of $44.08 \mathrm{~nm}$ and quite uniform size distribution (Fig. 1b). Their average particle size slightly increased up to $49.53 \mathrm{~nm}$ upon GQDs immobilization (Fig. 1d, e). $\mathrm{N}_{2}$ adsorption-desorption isotherms (Fig. 1f) also confirm that mesoporous silica nanoparticles were obtained, as they can be characterized with type IV isotherm, exhibiting a hysteresis loop. After immobilizing the GQDs onto MSNs, obtained GQDsMSNs maintained mesoporous structure; however, the $\mathrm{N}_{2}$ adsorption amount decreased sharply. Similarly, upon GQDs immobilization, the surface area (SSA) and pore volume decreased from 442.33 to $197.40 \mathrm{~m}^{2} / \mathrm{g}$ and from 1.06 to $0.65 \mathrm{~cm}^{3} / \mathrm{g}$. These results confirm indirectly that GQDs were successfully immobilized onto MSNs. The broad diffraction peak at 15-30 $2 \theta^{\circ}$ indicates that prepared MSNs have an amorphous structure (Fig. 1c). The XRD pattern does not reveal any structural changes of MSNs upon GQDs immobilization. The mesoporous structure with high surface area and pore volume indicate that prepared GQDs-MSNs have a great potential for drug-loading efficiency.

The successful preparation of GQDs, MSNs, and GQDs-MSNs nanocomposite nanoparticles, as well as their successive modifications and loading with DOX are revealed and confirmed with the FTIR spectroscopy (Fig. 2). FTIR spectrum of GQDs (Fig. 2a) is featured IR absorption bands typical for graphene derived structures, namely (i) C-O-C stretching (1109 and $\left.1254 \mathrm{~cm}^{-1}\right)$; (ii) phenolic $-\mathrm{OH}$ stretching $\left(1395 \mathrm{~cm}^{-1}\right)$; (iii) $\mathrm{C}-\mathrm{C}\left(1457 \mathrm{~cm}^{-1}\right)$; (iv) aromatic $\mathrm{C}=\mathrm{C}\left(1572 \mathrm{~cm}^{-1}\right)$, but also $\mathrm{C}=\mathrm{O}$ stretching $\left(1726 \mathrm{~cm}^{-1}\right)$ of carboxylic groups (Yao et al. 2017a, b; Yao et al. 2017a, b). In the 2800-3000 $\mathrm{cm}^{-1}$ region, the C-H-related bands are exhibited: aldehyde doublet, methylene asymmetric stretching, and methyl asymmetric stretching at $2829 \mathrm{~cm}^{-1}, 2845 \mathrm{~cm}^{-1}$, and $2940 \mathrm{~cm}^{-1}$, respectively. In turn, bands observed in the range of $3000-4000 \mathrm{~cm}^{-1}$ could be attributed to hydroxyl and amine groups. The presence of - $\mathrm{NH}$ groups is strictly related to remaining impurities of thiourea applied during the synthesis process. This could be additionally confirmed by the presence of C-N at 1329 and $1179 \mathrm{~cm}^{-1}$, as well as C-S at $690-$ and $650 \mathrm{~cm}^{-1}$-stretching vibrations. Therefore, these IR spectra confirm that hydrothermally prepared GQDs are rich in oxygen-containing functional groups, thus have a potential to react further with $\mathrm{NH}_{2}$-MSNs. In case of MSNs-based samples (Fig. 2b-f), all the spectra are featured by presence of Si-O-Si bending vibration, Si-O symmetric stretching, Si-OH symmetric stretching, and Si-O-Si asymmetric stretching in range of 680 $695 \mathrm{~cm}^{-1}, 780-795 \mathrm{~cm}^{-1}, 945-990 \mathrm{~cm}^{-1}$, and 1075$1090 \mathrm{~cm}^{-1}$, respectively. Moreover, one can notice scissor-bending vibrations of adsorbed molecular water in range of 1615-1645 $\mathrm{cm}^{-1}$, as well as H-bonded silanol -OH groups in range of $3365-3395 \mathrm{~cm}^{-1}$ and $3635-3650 \mathrm{~cm}^{-1}$. From the spectrum of bare MSNs (Fig. 2b), one can notice the presence of asymmetric $\mathrm{CH}_{2}$ and $-\mathrm{CH}_{3}$ stretching vibrations at 2904 and $2979 \mathrm{~cm}^{-1}$, respectively. The presence of methylene and methyl groups could be related to remaining impurities like unreacted Si-precursor TEOS or surfactant $\mathrm{CTAB}$, used during the synthesis. In case of $\mathrm{NH}_{2}-\mathrm{MSNs}$ sample (Fig. 2d), one can observe intensity decrease of the $\mathrm{Si}-\mathrm{O}$ symmetric stretching vibration along with the disappearance of Si-OH symmetric stretching, which proves successful MSNs functionalization with aminosilane compound. However, the amine groups vibrations are overlapped by free or adsorbed $-\mathrm{OH}$ groups. Therefore, $-\mathrm{NH}_{2}$ presence is confirmed by the broadening of the band in range of $3050-3700 \mathrm{~cm}^{-1}$. Introduced amine groups were further used to covalently bind GQD to MSNs via amide bond (C-N), which is present in GQD@MSNs spectrum at $1695 \mathrm{~cm}^{-1}$ 

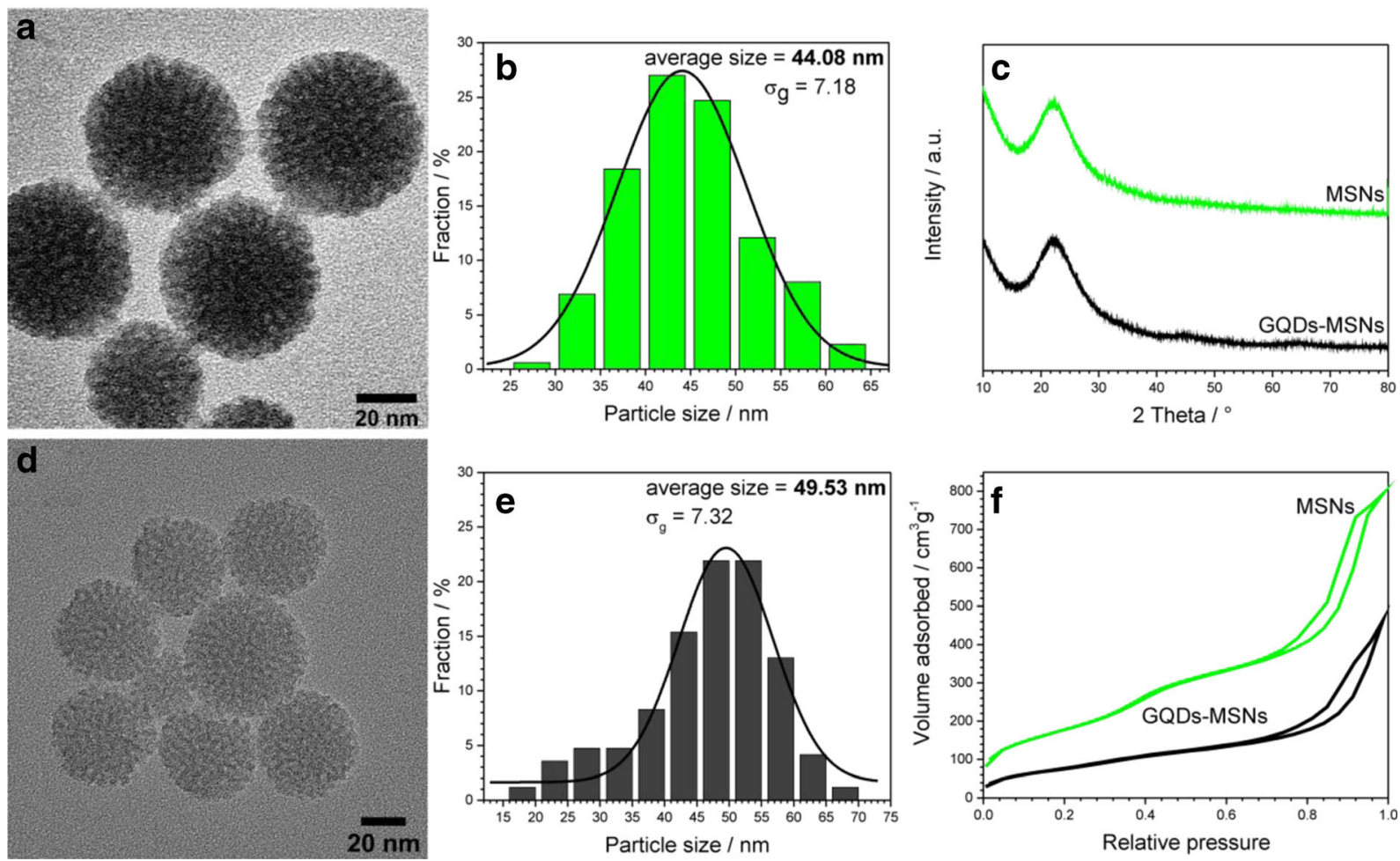

Fig. 1 TEM images and particle size distribution of prepared MSNs (a, b) and GQDs-MSNs nanocomposite nanoparticles (d, e); XRD patterns (c) and $\mathrm{N}_{2}$ adsorption/desorption isotherms (f)

(Fig. 2c), next to the bands typical for MSNs and GQD previously described. Finally, the adsorption of the doxorubicin onto MSNs was also investigated. Figure 2e shows the IR spectrum of DOX-MSNs, where the presence of drug is confirmed with the following bands: C-C stretching $\left(1413 \mathrm{~cm}^{-1}\right),-\mathrm{OH}$ bending $\left(1444 \mathrm{~cm}^{-1}\right)$, aromatic $\mathrm{C}=\mathrm{C}$ vibrations $(1516$ and $\left.1580 \mathrm{~cm}^{-1}\right)$, N-H bending $\left(1611 \mathrm{~cm}^{-1}\right)$, as well as $\mathrm{C}=\mathrm{O}$ stretching $\left(1726 \mathrm{~cm}^{-1}\right)$ of carboxylic groups. All of the doxorubicin-related bands can be also observed in the spectrum of DOX-GQDs-MSNs (Fig. 2f). The positions of the peaks are slightly up-shifted $\left(+5 \mathrm{~cm}^{-1}\right)$, and their intensities are decreased. Moreover, the contribution of typical bands for graphene structures diminishes upon DOX loading, which indicates the stronger interaction between GQDs and DOX molecules. Nevertheless, these results confirm successful formation of designed drug delivery system.

Due to the foreseen biomedical application of prepared GQDs-MSNs nanocomposite nanoparticles, they should form stable suspensions in water-based media. Therefore, the zeta potential was controlled at each stage of their preparation, as well as after surface functionalization and further doxorubicin drug attachment. The results are given in Table 1. Both prepared bare GQDs and MSNs exhibit moderately high zeta potential in water-based suspension and are negatively charged, -24.2 and $-30.0 \mathrm{mV}$, respectively. This is in agreement with reported isoelectric point and zeta potential of MSNs (Wu et al. 2013a) and of GQDs (Wu et al. 2013b). The negative charge is resultant of number of oxygen-containing functional groups at both GQDs and MSNs, in agreement with FTIR spectroscopy. After modifying with amino groups, the zeta potential of $\mathrm{NH}_{2}$-MSNs changed to the positive one with higher value of $+34.1 \mathrm{mV}$. In turn, after covalent immobilization of GQDs, the zeta potential remained positive and exhibited value of $+31.1 \mathrm{mV}$ for GQDs-MSNs. Hence, these results confirm successful functionalization and preparation of GQDs-MSNs nanocomposite nanoparticles. Their high zeta potential is an advantage, as they are considered to form relatively stable suspensions. Moreover, the positive charge of GQDs-MSNs will enable their efficient cellular uptake via the electrostatic interaction with negatively charged cell membranes (Frohlich 2012). 

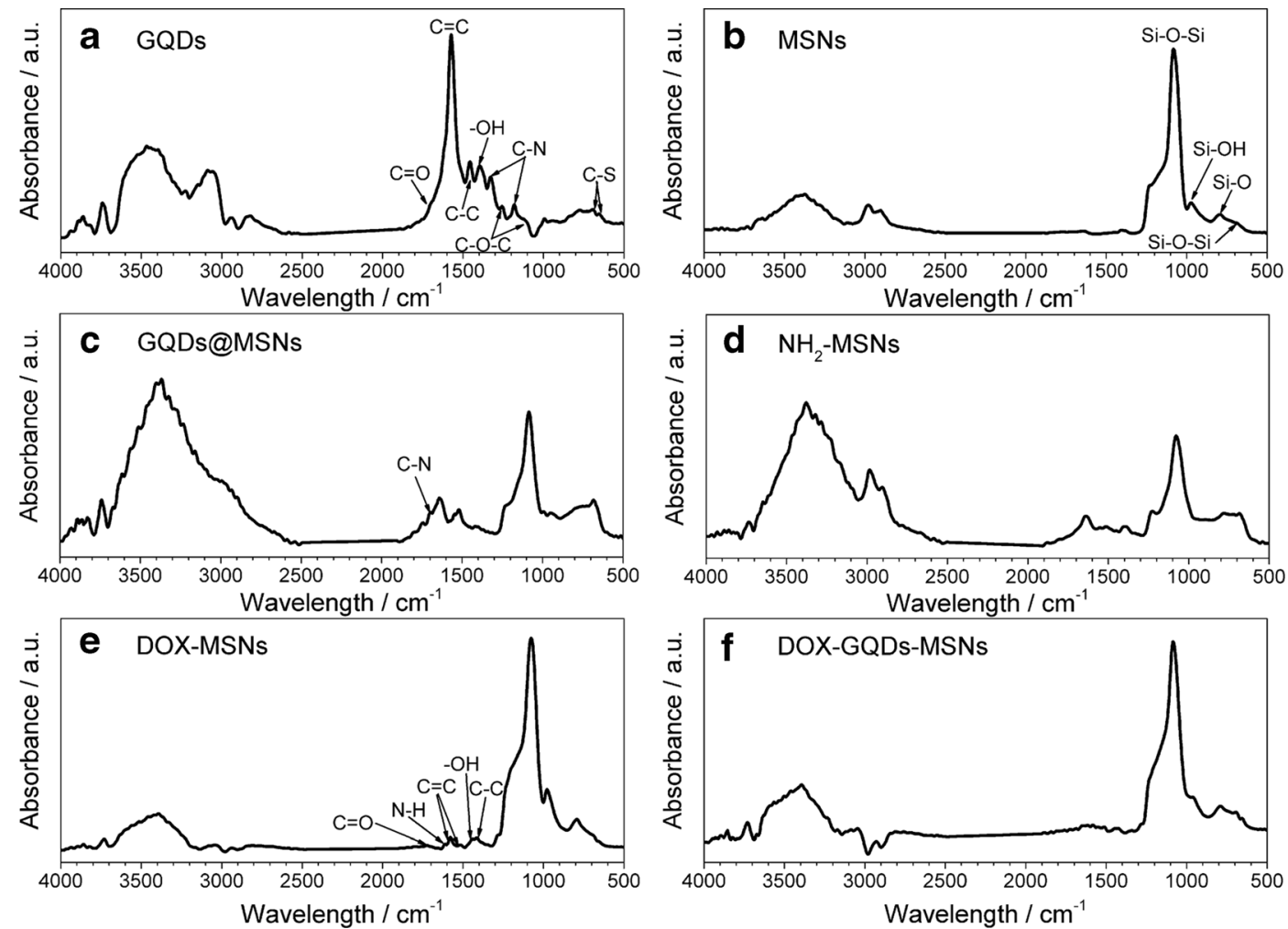

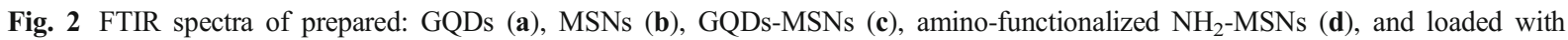
doxorubicin DOX-MSNs (e) and DOX-GQDs-MSNs (f)

Optical properties

Surface passivation with carbonyl groups and heteroatom doping, revealed by FTIR and XPS, find the reflection in the optical properties, as they change the electronic density of bulk semiconductor materials (Zhu et al. 2012; Permatasari et al. 2016). The UV-Vis absorption and photoluminescent (PL) excitation and emission properties were investigated using waterbased suspensions of both GQDs and GQDs-MSNs (Fig. 3). The PL quantum yield of GQDs for the emission at $440 \mathrm{~nm}$ was calculated to be $39.5 \%$. In the UV-

Table 1 Summary of the physicochemical properties: the average particle size $\left(d_{\text {TEM }}\right)$ and standard geometric deviation $\left(\sigma_{g}\right)$, the surface area $(\mathrm{SSA})$ and pore volume $(\mathrm{V})$, zeta potential $(\zeta)$ results

measured in water-based $\left(\mathrm{H}_{2} \mathrm{O}_{\mathrm{d}}\right)$ suspensions; DOX-loading and release efficiency (\% $\mathrm{DOX}_{\text {loaded }}$, loading capacity in $\mu \mathrm{g} \mathrm{DOX} /$ $1 \mathrm{mg}$ NPs, and release in \%)

\begin{tabular}{|c|c|c|c|c|}
\hline Sample name & $d_{(\mathrm{TEM})} / \sigma_{g}$ & $\mathrm{SSA}\left(\mathrm{cm}^{2} / \mathrm{g}\right) / \mathrm{V}\left(\mathrm{cm}^{3} / \mathrm{g}\right)$ & \multicolumn{2}{|c|}{ Zeta potential $(\mathrm{mV}) / \mathrm{std}$. dev. } \\
\hline MSNs & $44.08 / 7.18$ & $442.33 / 1.06$ & \multicolumn{2}{|c|}{$-30.0 / 0.3$} \\
\hline $\mathrm{NH}_{2}$-MSNs & - & - & \multicolumn{2}{|c|}{$+34.1 / 0.7$} \\
\hline GQDs & $3.65 / 0.81$ & - & \multicolumn{2}{|c|}{$-24.2 / 1.2$} \\
\hline GQDs-MSNs & $49.53 / 7.32$ & $197.40 / 0.65$ & \multicolumn{2}{|c|}{$+31.1 / 0.8$} \\
\hline DOX-MSNs & - & - & \multicolumn{2}{|c|}{$-6.9 / 1.6$} \\
\hline DOX-GQDs-MSNs & - & - & \multicolumn{2}{|c|}{$+30.8 / 1.8$} \\
\hline \multirow[t]{2}{*}{ Sample name } & \multirow[t]{2}{*}{$\%$ DOX $_{\text {loaded }}$} & \multirow[t]{2}{*}{ Loading capacity ( $\mu \mathrm{g}$ DOX/1 mg NPs) } & \multicolumn{2}{|c|}{ Release at pH 5.0 after 48 h (\%) } \\
\hline & & & $37^{\circ} \mathrm{C}$ & $50{ }^{\circ} \mathrm{C}$ \\
\hline DOX-MSNs & 78.88 & 399.4 & 55.33 & 89.12 \\
\hline DOX-GQDs-MSNs & 53.93 & 269.7 & 26.96 & 28.42 \\
\hline
\end{tabular}


Vis absorbance spectra of GQDs, a strong absorption peak at $330 \mathrm{~nm}$ is observed, which corresponds to the $\mathrm{n}-\pi^{*}$ transition of the conjugated $\mathrm{C}=\mathrm{O}$ bonds in carbon$\mathrm{yl}$ groups present at the surface and edges of prepared GQDs and of possible $\mathrm{C}=\mathrm{N}$ bonds (Qu et al. 2014), the presence of which was revealed by XPS and FTIR. By the contrast, the prepared GQDs-MSNs suspension reveal high absorbance background of decreasing intensity with increasing wavelength. The PL excitation spectra (Fig. 3b, d) are consistent with the absorbance spectra, as their maxima overlap with the absorbance maxima. The highest PL emission intensity is obtained for the excitation between 340 and $360 \mathrm{~nm}$ (Fig. 3a, c). GQDs and GQDs-MSNs solutions exhibit the blue PL emission with the maximum peak located at around $440 \mathrm{~nm}$ under UV excitation from 320 to $360 \mathrm{~nm}$. GQDs-MSNs solution reveals significantly lower PL emission intensity. Maximum intensity of PL emission is observed under excitation wavelength of 360 and $340 \mathrm{~nm}$ for GQDs and GQDs-MSNs, respectively. The Stokes shift is therefore about $80 \mathrm{~nm}$ for GQDs and $95 \mathrm{~nm}$ for GQDs-MSNs. Under excitation above 400 to $550 \mathrm{~nm}$ the maximum PL emission peak shifts towards longer wavelengths from 440 to about $600 \mathrm{~nm}$, along with the intensity decrease. Therefore, the PL emission of prepared GQDs and GQDs-MSNs can be considered as excitation-dependent, with three main PL regions (blue emission for the excitation up to $400 \mathrm{~nm}$, green emission for the excitation below $480 \mathrm{~nm}$, and red emission for the excitation below up to $550 \mathrm{~nm}$ ). This effect will be particularly presented in the bioimaging studies. The excitation-dependent PL emission has been previously observed in GQDs, and it was related to the presence of GQDs with different sizes or conjugated $\mathrm{sp}^{2}$ domains (Sk et al. 2014). Therefore, we can conclude that the blue emission results from the chromophores related to $\mathrm{C}=\mathrm{O}$ bonds (observed in absorbance spectrum). The green and red emission therefore must have an another chromophore origin, possibly related to the $\mathrm{C}=\mathrm{N}$ bonds ( $\mathrm{Qu}$ et al. 2014), but also due to the presence of different sized GQDs (Sk et al. 2014). This is also well reflected in the PL excitation spectra for the emission above $420 \mathrm{~nm}$, which reveal more than one excitation maximum, dominating one at $330 \mathrm{~nm}$ (as in absorbance spectra), but also excitation peaks about $370 \mathrm{~nm}$, in the dominating peak shoulder. This second excitation contribution is more pronounced (increasing for the emissions at longer wavelengths) in case of GQDsMSNs. This suggests the scenario of forming clusters of GQDs at the MSNs surface, which retain the fundamental optical properties of the individual GQDs, however with an influence on their PL emission intensity in the water-based solutions. Effect of the clustering of QDs on their optical properties and resulting application as bioimaging labels has been investigated, e.g., for $\mathrm{CdSe} / \mathrm{CdS}$ quantum dots and quantum rods (Rafipoor et al. 2015) or more recently in $\mathrm{ZnAgInSe} / \mathrm{ZnS}$ QDs (Deng et al. 2017).

\section{DOX-loading and release profile}

To study the anticancer drug-loading and release efficiency, doxorubicin hydrochloride was non-covalently loaded onto prepared GQDs-MSNs nanocomposite nanoparticles, as well as onto un-modified MSNs as a control drug carrier. The advantage of the adsorption interaction due to the high surface area of mesoporous carrier, as well as electrostatic attraction was exploited for this purpose. The DOX-loading efficiency (\% DOX $_{\text {loaded }}$ ) was estimated spectrophotometrically to be 53.93 (which corresponds to the loading capacity of $269.7 \mu \mathrm{g} / \mathrm{mg}$ ) and $78.88 \%$ (which corresponds to the loading capacity of $399.4 \mu \mathrm{g} / \mathrm{mg}$ ) for GQDs-MSNs and MSNs, respectively. As expected, the drug-loading efficiency was higher in case of control un-modified MSNs, what can be assigned to their high surface area and pore volume, but also to the efficient electrostatic attraction between positively charged DOX molecules and negatively charged MSNs. When considering DOX-loading onto GQDs-MSNs, the drug-loading efficiency was reduced, firstly by their lower specific surface area and pore volume, which were blocked by the GQDs immobilized onto MSNs. Secondly, the drug-loading efficiency through the electrostatic attraction is unfavorable to take place owing to the positive charge of GQDsMSNs. Results of the zeta potential measurements (see, Table 1) show that due to the DOX-loading the electrophoretic potential of DOX-GQD@MSN suspension does not change significantly and remain positive (+ $30.8 \mathrm{mV}$ ). Whereas for DOX-MSNs, the significant zeta potential decrease is observed; however, it remains negative value $(-6.9 \mathrm{mV})$. These results indicate that waterbased suspensions of prepared DOX-GQDs-MSNs exhibit better stability than DOX-MSNs; hence, these nanocomposite nanoparticles can be considered as more preferred carrier for drug delivery application.

The drug release behavior was investigated in response to different $\mathrm{pH}(4.5,5.0,6.5,7.4)$ and 
Fig. 3 Optical properties of prepared GQDs (a, b) and GQDsMSNs nanocomposite nanoparticles $(\mathbf{c}, \mathbf{d})$ : excitationdependent PL emission (Em) spectra and absorbance (Abs) spectra $(\mathbf{a}, \mathbf{c})$; PL excitation (Exc) spectra $(\mathbf{b}, \mathbf{d})$
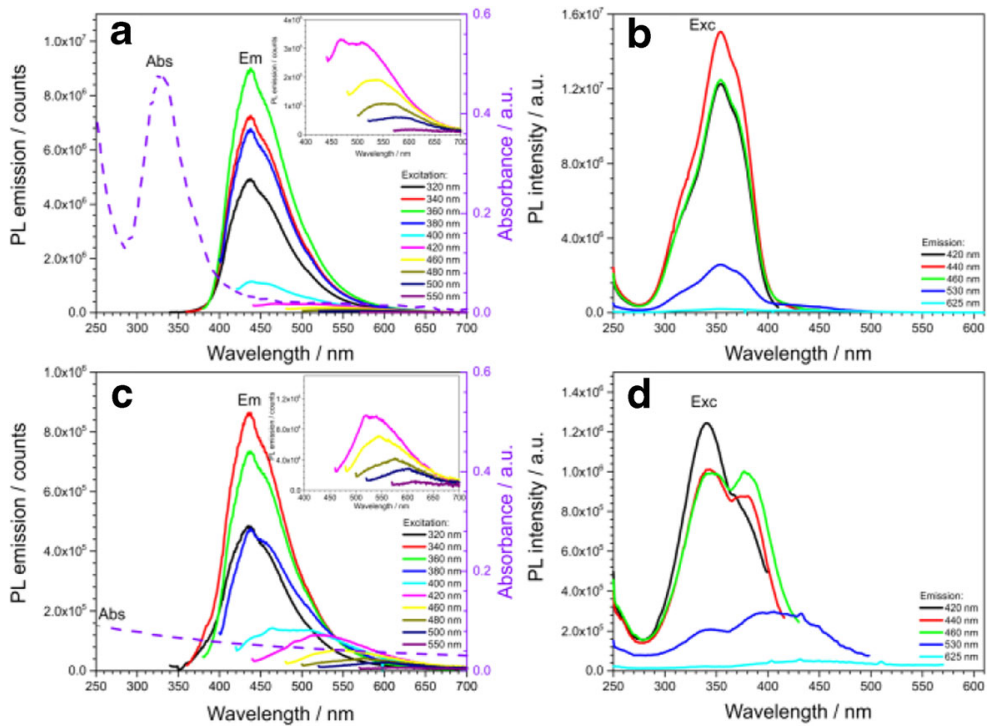

temperature $\left(37\right.$ and $50{ }^{\circ} \mathrm{C}$ ) in PBS-based release solution. The acidic and neutral $\mathrm{pHs}$ of a release solution were chosen as they reflect best the tumor environment (endosomes and lysosomes of cancer cells) and a physiological environment in normal tissue, respectively (Upreti et al. 2013). Figure 4 shows the cumulative DOX release from DOX-GQDs-MSNs and DOXMSNs under different conditions. All profiles exhibit gradual release over the time, without the rapid initial DOX release. The release of DOX was fastest in the medium with $\mathrm{pH} 4.5$ and 5.0 and at $50{ }^{\circ} \mathrm{C}$ for both prepared carriers. Obviously, the release of DOX was more efficient in case of DOX-MSNs, and 90.02 and $89.12 \%$ of loaded drug was released within $48 \mathrm{~h}$ at $50{ }^{\circ} \mathrm{C}$, respectively at $\mathrm{pH} 4.5$ and 5.0. By contrast, DOX-GQDs-MSNs exhibited slower release rate, and 36.55 and $28.42 \%$ of loaded drug was released within $48 \mathrm{~h}$ at $50^{\circ} \mathrm{C}$, respectively at $\mathrm{pH} 4.5$ and 5.0. This can be ascribed to the lower drug-loading efficiency, but also to the stronger type of interaction between DOX molecules and GQDs-MSNs, as shown with FTIR analysis, in comparison to the weak adsorption and electrostatic attraction in case of DOX-MSNs. Therefore, also the temperature effect was more significant in case of DOXMSNs, where the temperature increase could weaken the interaction and facilitates DOX molecules release. It can be also concluded that both DOX-GQDs-MSNs and DOX-MSNs carrier exhibit a $\mathrm{pH}$-dependent drug release behavior, as the DOX release can be triggered by acidic conditions, due to the weakening of the interaction between DOX molecules and GQDs-MSNs and
MSNs. Hence, the drug delivery and release in cancer cells could be enhanced by the use of proposed nanocarriers, and further could result in diminishing the side effects of DOX. Although the low DOXrelease efficiency is observed for DOX-GQDs-MSNs, the released amount of drug is enough to trigger the cytotoxic effects and what will be further presented.

In vitro cytotoxicity

In order to estimate the cytotoxicity of GQDs, MSNs as well as GQDs-MSNs nanocomposite nanoparticles, the WST-1 assay was firstly performed. This colorimetric assay is based on the cleavage of a tetrazolium salt by mitochondrial dehydrogenases forming formazan in viable cells. Therefore, the greater the amount of formazan is produced following the addition of WST-1, the greater the number of metabolically active viable cells. Figure 5a, b shows the cell viability of cervical cancer cells (HeLa) and normal human fibroblasts (MSU1.1) after incubation for $24 \mathrm{~h}$ with a series of concentrations of GQDs, MSNs, and GQDs-MSNs, also loaded with doxorubicin. The results show that GQDs, MSNs, and GQDs-MSNs only slightly affect the viability of both cells type, which remained above $90 \%$. Only the highest concentration $(250 \mu \mathrm{g} / \mathrm{ml})$ of nanoparticles was more deleterious to cells; however, even in this case, the viability remained above $80 \%$.

The loading of doxorubicin onto MSNs or GQDsMSNs generally results in a decrease of cells viability in a concentration-dependent manner. In case of HeLa 
Fig. 4 The release of doxorubicin (DOX) from MSNs and GQDs-MSNs at different temperatures $\left(37\right.$ and $\left.50{ }^{\circ} \mathrm{C}\right)$ and different $\mathrm{pH}$ values

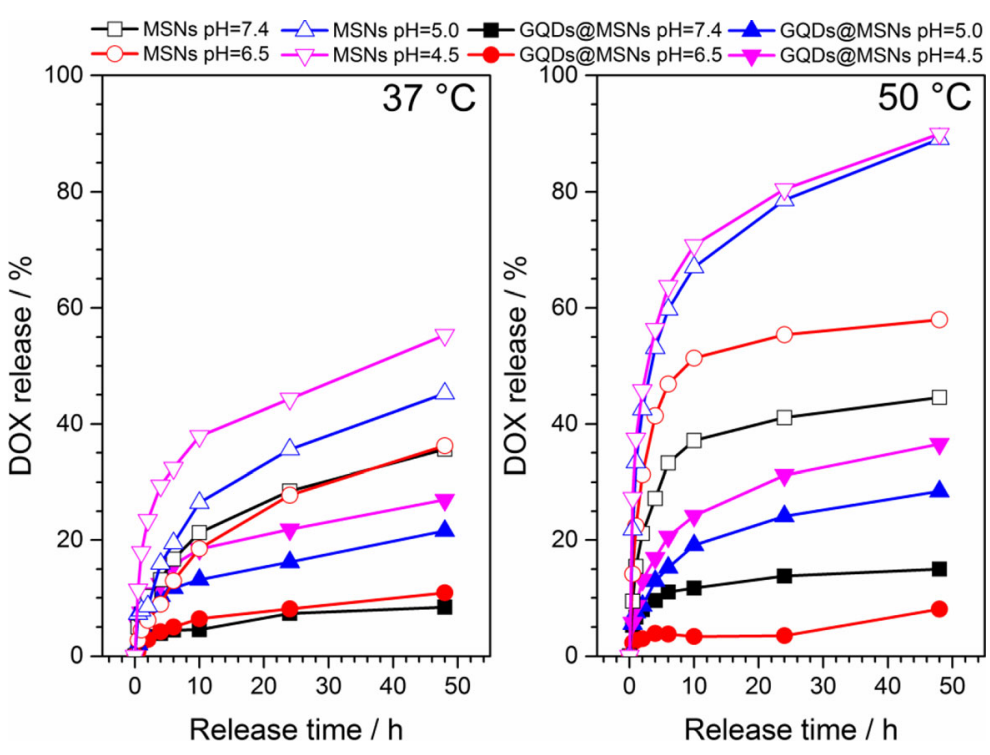

cells, the decrease after incubation with DOX-MSNs proceeds very rapidly from the $25 \mu \mathrm{g} / \mathrm{ml}$ nanoparticles concentration, and more than $80 \%$ of cells are indicated as death. Whereas, for DOX-GQDs-MSNs-treated cells a marked decrease in viability is observed at higher concentration of $250 \mu \mathrm{g} / \mathrm{ml}$. This is in agreement with the presented above DOX-release profile, showing that the amount of released DOX from DOX-GQDs-MSNs is much lower than from DOX-MSNs, therefore justifying the need for higher nanocarrier concentration in this case, but not a higher dose of DOX itself.

In comparison, for human fibroblasts, the viability decrease is milder; however, for DOX-MSNs-treated cells, the $50 \%$ inhibition of the cell viability is observed already at the concentration of $10 \mu \mathrm{g} / \mathrm{ml}$ and then decline gradually. The cytotoxicity of DOX-GQDs-MSNs initially seems to be lower, but ultimately at higher concentrations (from $50 \mu \mathrm{g} / \mathrm{ml}$ ) reaches higher value than for DOX-MSNs.

The WST-1 results on the cytotoxicity of prepared nanomaterials, reflecting the metabolic activity of cells, were then compared to plasma membrane integrity of cells, indicated by the intracellular esterase activity with the LIVE/DEAD assay (Fig. 5c, d). Here, two dyes were used, which quickly discriminates live from dead cells by simultaneous staining with green-fluorescent calcein-AM, indicating intracellular esterase activity and red-fluorescent ethidium homodimer-1, indicating a loss of plasma membrane integrity. In addition, the fluorescence images acquired with the InCell Apparatus (Fig. 5e) give an overview on the cell viability but also proliferation. In case of nanoparticles without doxorubicin, obtained results are comparable with those from WST-1 assay. The only difference is observed in case of fibroblasts, where at the highest concentration of MSNs and GQDs-MSNs the viability does not exceed $70 \%$. Moreover, evaluation of DOX-MSNs and DOX-GQDsMSNs cytotoxicity based on the cell membrane integrity analysis shows that these drug carriers are not as cells deleterious as it was presented above with the metabolic activity analysis. It is presented (particularly for fibroblasts), that even at the highest applied doses of $250 \mu \mathrm{g} / \mathrm{ml}$, the viability was maintained at 80 and $60 \%$ for DOX-MSNs and DOX-GQDs-MSNs, respectively. However, as presented in Fig. 5e, the treatment with DOX-loaded nanoparticles has a strong effect on the cell proliferation, which is particularly the case of DOXMSNs, in comparison to control non-treated cells. Therefore, found with these two assays differences in the cytotoxicity of prepared nanoparticles, arise from the fact that different aspects of cell functions were analyzed, cell viability and cell vitality, by live/dead assay and WST-1, respectively (Kwolek-Mirek and ZadragTecza 2014). However, both are required for the estimation of the physiological state of a cell after exposure to various types of stressors, including nanoparticles. Whereas, the fluorescent staining distinguishes live from dead cells, the WST-1 assay gives the information about their ability to reproduction. Hence, the lower number of living cells upon DOX-MSNs and DOXGQDs-MSNs indicates the action of successfully delivered and released doxorubicin. Further, both assays 

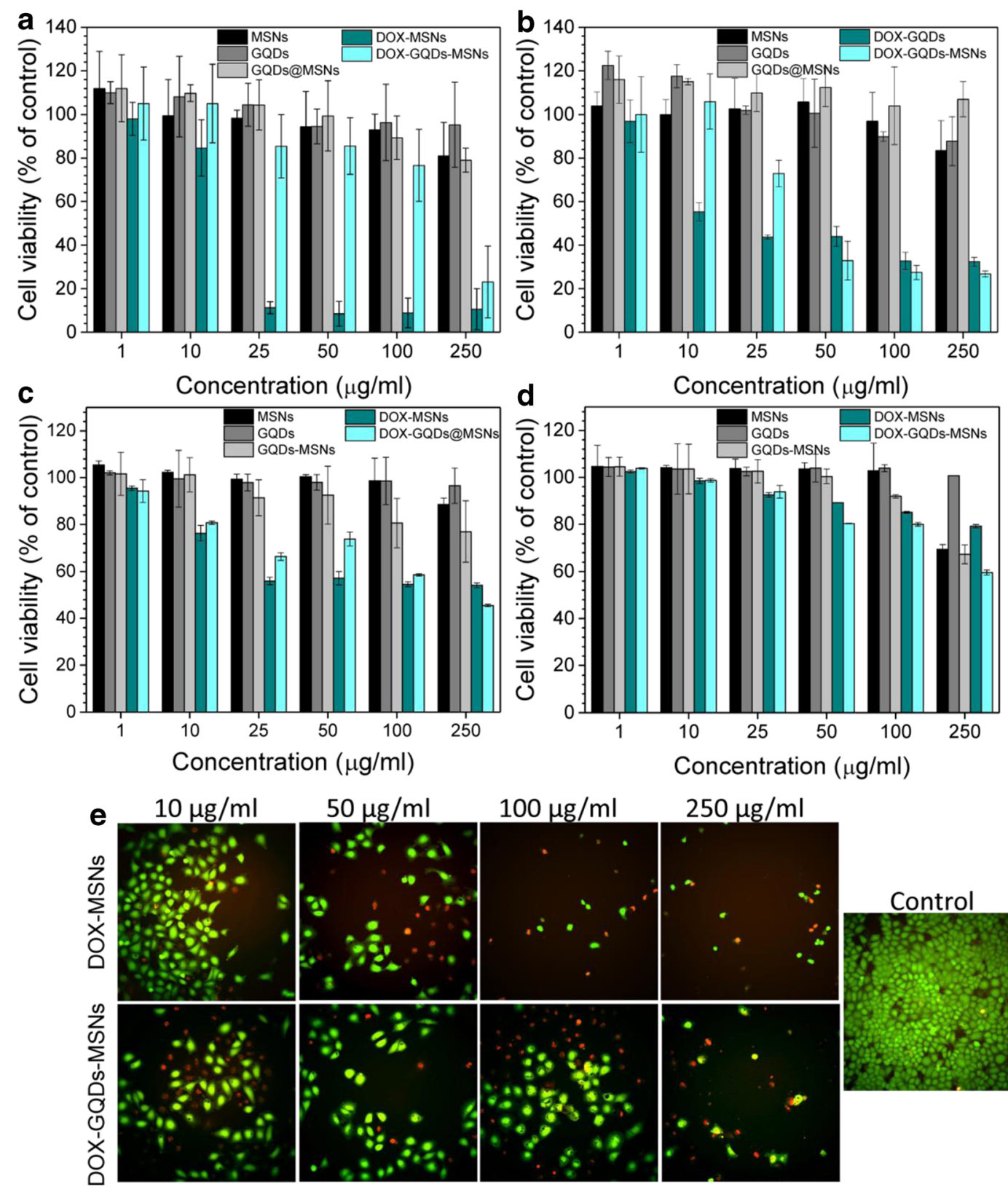

$100 \mu \mathrm{g} / \mathrm{ml}$
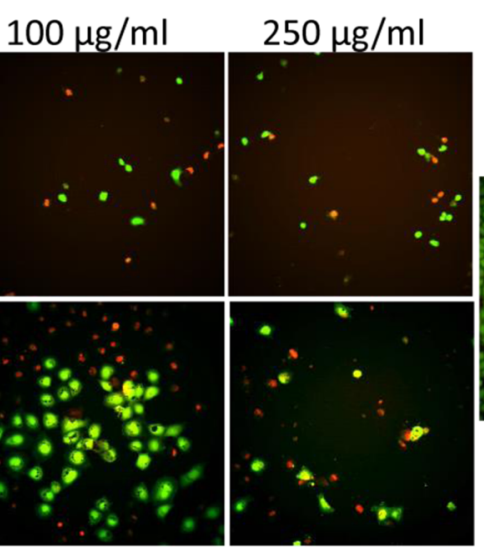

Fig. 5 Cell viability of HeLa cells (a, c) and MSU1.1 fibroblasts (b, d) after $24 \mathrm{~h}$ of incubation with different concentration of MSNs, GQDs, GQDs-MSNs, and loaded with drug DOX-MSNs and DOX-GQDs-MSNs, investigated with WST-1 assay $(\mathbf{a}, \mathbf{b})$ and Live/Dead assay kit and analyzed by InCell Analyzer apparatus; representative fluorescence images showing the treatment efficacy of DOX-MSNs and DOX-GQDs-MSNs on the HeLa cells viability and proliferation using calcein-AM and EthD-1 fluorescence staining (e). Cells with green fluorescence are considered as viable cells, whereas those with red fluorescence are considered as dead cells

Intracellular localization and uptake mechanism of GQDs-MSNs

The use of GQDs with their PL properties allows to monitor cellular uptake and distribution of GQDs- confirmed that conjugation of biocompatible GQDs with nontoxic MSNs, leads to the creation of lowcytotoxic GQDs-MSNs nanocomposite nanoparticles, making them particularly good candidates for drugdelivery system. 
MSNs nanocomposite nanoparticles directly. Moreover, the GQDs entrapment onto mesoporous silica nanoparticles structure resulted in the increasing fluorescent signal, especially in the red channel in comparison to bare GQDs (Fig. S3 in ESM). The intensification of obtained fluorescence signal could be related with immobilization and aggregation of GQDs onto MSNs, of what was previously suggested (Rafipoor et al. 2015; Deng et al. 2017), but also to their more effective penetration into cells, enabled by their preferred positive surface charge for the interaction with negatively charged cell membrane (Frohlich 2012). To demonstrate the fluorescence imaging performance of GQDs-MSNs nanocomposite nanoparticles, the in vitro cellular uptake experiments were performed using HeLa cells. Cells were exposed to $50 \mu \mathrm{g} / \mathrm{ml}$ of nanocomposite nanoparticles for $4 \mathrm{~h}$ and afterwards analyzed by CLSM. The results are presented in Fig. 6. Here, the excitationdependent PL behavior of the GQDs can be observed. Confocal images of HeLa cells incubated with nanoparticles upon $405 \mathrm{~nm}$ excitation exhibit the fluorescence emission in blue region, which could be observed as signals inside the cells. When the excitation light changes to $488 \mathrm{~nm}$, green fluorescence is observed; finally, upon 559 excitation, the red fluorescence is emitted. A similar effect was observed by Zhu et al. (Zhu et al. 2011) for GQDs, which however emitted green and yellow fluorescence. According to the merged images, GQDs-MSNs after $4 \mathrm{~h}$ of co-incubation were internalized by HeLa cells and localized in the cytoplasm. The internalization was clearly confirmed in the $3 \mathrm{D}$ images (Fig. 6b). It has been already proved that GQDs can be easily internalized into cells (Markovic et al. 2012; Yuan et al. 2014; Schroeder et al. 2016). Likewise, Lu et al. (Lu et al. 2007) demonstrated that MSNs nanoparticles are readily endocytosed by cells and usually enter cells in an energy-dependent manner.

In order to evaluate the exact uptake mechanism of GQDs-MSNs, the inhibition of selected routes were performed. Results given in Fig. S5 (ESM) indicate that by lowering the temperature to $4{ }^{\circ} \mathrm{C}$, the energy-dependent mechanisms is blocked. This is observed by lacking signal in cells, is comparison to control HeLa cells treated with $20 \mu \mathrm{g} / \mathrm{ml}$ GQDs-MSNs for $3 \mathrm{~h}$ at $37{ }^{\circ} \mathrm{C}$, where the signal in cells is clear. Therefore, the endocytosis is considered as an exact uptake mechanism of studied nanoparticles. However, by using suitable inhibitors, different types of endocytosis were further investigated. Chlorpromazine is reported to specifically inhibit clathrin-dependent endocytosis (CDE), while methyl- $\beta$ cyclodextrin has been extensively used to inhibit clathrinindependent endocytosis (CIE), especially in caveolae/ lipid raft-mediated endocytosis (Le et al. 2002; Parton and Richards 2003, dos Santos et al. 2011). Wortmannin, in turn, as covalent inhibitor of phosphoinositide 3kinases (PI3Ks), plays a role in inhibition of micropinocytosis (Araki et al. 1996; Rupper et al. 2001). As can be seen in Fig. S5, chlorpromazine showed no inhibitory capacity in HeLa cells on studied nanoparticles uptake. Contrary to chlorpromazine, the $\mathrm{M} \beta \mathrm{CD}$ inhibited the uptake of GQDs-MSNs into cells completely, suggesting the CIE as a main route of these nanoparticles internalization. Similar results were presented by Ekkapongpisit et al. (Ekkapongpisit et al. 2012), contrary to Hao et al. (Hao et al. 2012), who indicated that spherical mesoporous silica nanoparticles are internalized rather via the clathrin-mediated pathway. Interestingly, in our study, the wortmannin inhibits nanoparticles internalization partially, indicating that maybe larger nanoparticles or nanoparticles aggregates are internalized by micropinocytosis (Meng et al. 2011; Vollrath et al. 2013).

Additionally, to confirm that the uptake of GQDsMSNs nanocomposites nanoparticles is mediated through the endosome-lysosomal mechanism, TEM analysis was performed (Fig. 7). Obviously, the GQDs-MSNs are uptaken by HeLa cells via their encapsulation into vesicular compartments. The beginning of the uptake occurs by the initiation of plasma membrane invagination, then the nanocomposite nanoparticles are transported to the early endosomes (Fig. 7a), and finally, they are found to be clumped in lysosomes (Fig.7c). Moreover, as presented in magnified images, GQDs-MSNs maintain the spherical and porous morphology. The abovementioned results indicated that the GQDs-MSNs possess both strong enough fluorescence emission and internalization ability, what makes them a promising material for bioimaging and biolabeling.

Intracellular distribution of doxorubicin

Finally, the cell uptake and DOX-MSNs and DOXGQDs-MSNs and intracellular DOX release after 3 and $24 \mathrm{~h}$ of incubation with HeLa cells was also monitored with CLSM. As presented in the Fig. 8, after $3 \mathrm{~h}$ of incubation red fluorescence signals from DOX molecules in the DOX-MSNs and GQDs-MSNs nanoparticles can be observed mainly in the cytoplasm, suggesting the endocytosis of the nanoparticles by HeLa cells 

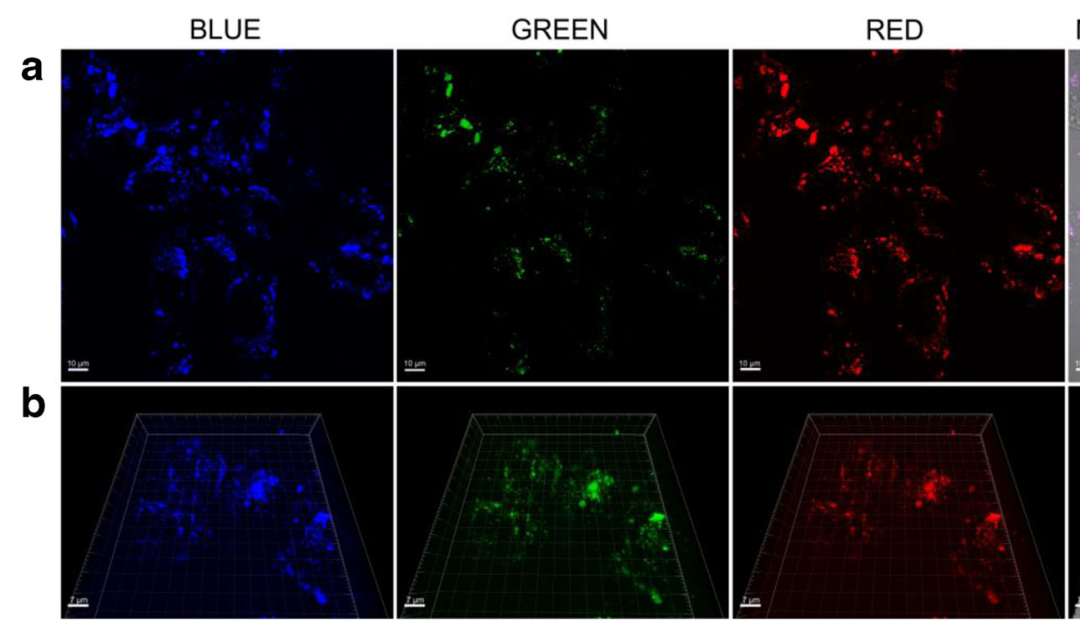

MERGE + BRIGHT FIELD

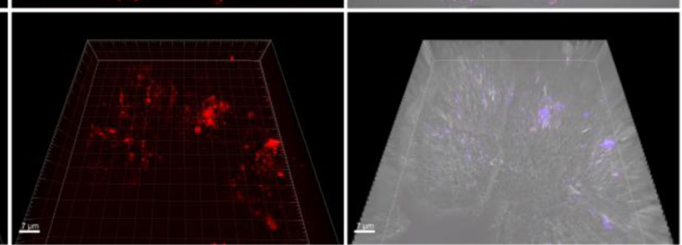

Fig. 6 2D (a) and 3D (b) CLSM imaging and intracellular localization of GQDs-MSNs in HeLa cells after $4 \mathrm{~h}$ of incubation $(50 \mu \mathrm{g} / \mathrm{ml})$. Channels blue, green, and red represent the

and DOX release. The observed bright red dots (marked with arrows in Fig. 8) are related to the aggregated carriers, whereas the homogenously spread red fluorescence signal throughout the cytoplasm is related to DOX molecules, which have been released from the nanoparticles within the acidic endosome and have diffused into the cytoplasm. Regarding the DOX-GQDs-MSNs, the use of fluorescent GQDs enables to monitor simultaneously the uptake of the DOX-GQDs-MSNs through channels corresponding to GQDs (mainly blue) and to DOX (red). This is consistent with the imaging results for HeLa cells treated with un-loaded GQD@MSNs presented in Fig. 6. Further incubation up to $24 \mathrm{~h}$ with higher dose of DOX-GQDs-MSNs $(200 \mu \mathrm{g} / \mathrm{ml})$ resulted fluorescence of GQDs in GQDs-MSNs at the excitation $405 \mathrm{~nm}$, $488 \mathrm{~nm}$, and $559 \mathrm{~nm}$, respectively (laser power of $0.5 \%$ )

in a marked increase in the releasing of DOX as observed with stronger fluorescent signal (Fig. S6), and these results are in accordance with the DOX-loading and release efficiency. Moreover, cytotoxicity studies indicated that the cytotoxic effect of DOX-GQDsMSNs is observed for doses above $100 \mu \mathrm{g} / \mathrm{ml}$ in case of HeLa cells. Therefore, in case of DOX-GQDs-MSNs, use of higher doses is necessary to induce effects similar to those caused by DOX-MSNs.

In order to investigate the colocalization of the GQDsMSNs nanocomposite nanoparticles with DOX, the analysis was performed using the Coloc2 tool and the Pearson's correlation coefficients were found. These coefficients are 0.77 and 0.44 for cells incubated with the
Fig. 7 TEM images of HeLa cells treated with GQDs-MSNs showing the cellular uptake and intracellular translocation: a endocytosis of nanoparticles through plasma membrane invagination (marked with a square), then GQDs-MSNs are sequestered and dispersed in early endosomes, $\mathbf{b}$ magnified image of early endosome with nanoparticles, c GQDs-MSNs condensed in late endosome/ lysosome, $\mathbf{d}$ magnified image of nanoparticles in late endosome/ lysosome
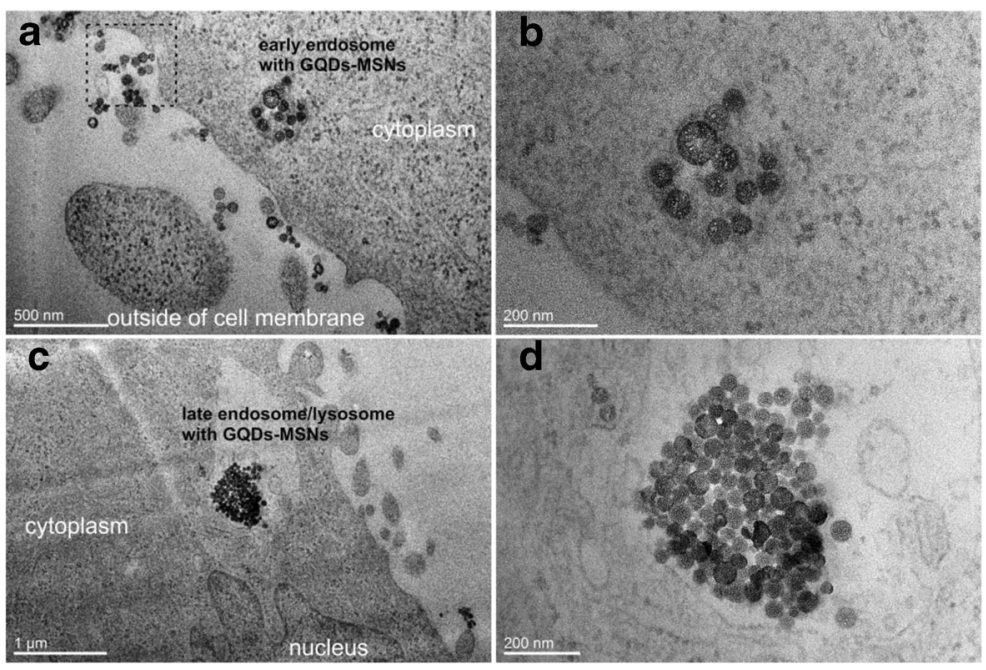
Fig. 8 Intracellular distribution of doxorubicin (DOX) delivered and released from DOX-MSNs (upper panel) and DOX-GQDsMSNs (lower panel) in HeLa cells after 3 and $24 \mathrm{~h}$ of incubation with HeLa cells. Channel blue (excitation $405 \mathrm{~nm}$ ) represents the fluorescence of DAPI-stained nuclei and the fluorescence of GQDs in DOX-GQDs-MSNs, while channel red represents the fluorescence of DOX (excitation $559 \mathrm{~nm}$ ). Scale bars, $10 \mu \mathrm{m}$

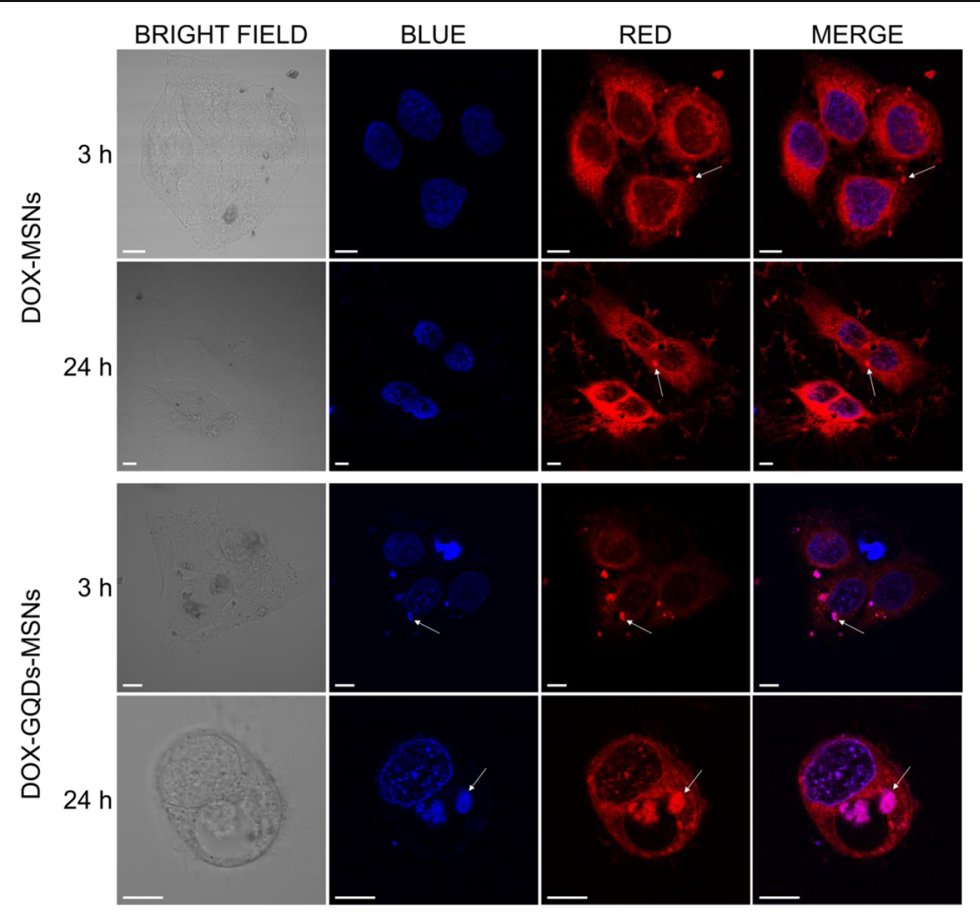

$20 \mu \mathrm{g} / \mathrm{ml}$ of DOX-GQDs-MSNs for 3 and $24 \mathrm{~h}$, respectively. In case of the cell exposure to $200 \mu \mathrm{g} / \mathrm{ml}$ of DOXGQDs-MSNs for 3 and $24 \mathrm{~h}$, found coefficients were 0.39 and 0.37 , respectively. These results indicate that colocalization occurs and is more significant in case of shorter time of incubation, whereas longer time of incubation results in the decreased colocalization coefficient. This confirms the time-dependent release of DOX from the GQDs-MSNs nanocomposite nanoparticles.

Summarizing, DOX-GQDs-MSNs nanocomposite nanoparticles are found to be efficient in DOX delivery via their internalization in HeLa cancer cells but also allow for simultaneous real-time optical tracking of the DOX during its delivery and release.

\section{Conclusions}

In this study, we reported on the successful preparation of GQDs-MSNs nanocomposite nanoparticles as an efficient intracellular drug delivery system, but also simultaneously, as fluorescent agent for the optical imaging. Prepared GQDs-MSNs with an average particle size is below $50 \mathrm{~nm}$ and high-positive zeta potential form stable suspensions exhibiting excitationdependent PL behavior. Moreover, they can be easily loaded with doxorubicin chosen as a model drug and show the $\mathrm{pH}-$ and temperature-dependent doxorubicin release behavior, due to weakening of the interaction between DOX molecules and GQDs-MSNs. The cytotoxicity assays confirmed that the conjugation of biocompatible GQDs with nontoxic MSNs, leads to the creation of GQDs-MSNs nanocomposite nanoparticles with negligible cytotoxicity, which may serve as a potential drug nanocarriers. Moreover, these assays also confirmed the therapeutic action of delivered and released doxorubicin. Further, the in vitro optical imaging efficacy of cells with GQDs-MSNs, resultant of their cellular internalization via caveloae/lipid raft-mediated endocytosis in HeLa cells, was proven with CLSM and confirmed with TEM imaging. The GQDs entrapment onto mesoporous silica nanoparticles structure resulted in the increasing fluorescent signal in comparison to bare GQDs, which could be related with the immobilization and aggregation effect, but also with their surface charge-related more effective intracellular penetration. Moreover, proposed GQDs-MSNs drug delivery systems enabled the simultaneous real-time optical tracking of the drug during its delivery and release, but also the monitoring of the penetration of the nanoparticles itself. These results indicate therefore that the GQDs-MSNs possess both strong enough fluorescence emission and internalization ability, what makes them a promising material for bioimaging and biolabeling. 
Acknowledgements Authors would like to thank Dr. Marcin Jarek and Dr. Mateusz Kempiński (Faculty of Physics, Adam Mickiewicz University in Poznań) for support during the XRD measurements and running XPS measurements, respectively.

Funding information This work was supported by the National Science Center, Poland (NCN) under research grant Miniatura 2017/01/X/ST5/00134. Partial support by the National Science Centre grant SONATA-BIS 6: 2016/22/E/ST3/00458 is also acknowledged. Teofil Jesionowski during the research was supported by the PUT research grant no. 03/32/DSPB/0806/2018.

\section{Compliance with ethical standards}

Conflict of interest The authors declare that they have no conflict of interest.

Open Access This article is distributed under the terms of the Creative Commons Attribution 4.0 International License (http:// creativecommons.org/licenses/by/4.0/), which permits unrestricted use, distribution, and reproduction in any medium, provided you give appropriate credit to the original author(s) and the source, provide a link to the Creative Commons license, and indicate if changes were made.

\section{References}

Araki N, Johnson MT, Swanson JA (1996) A role for phosphoinositide 3-kinase in the completion of macropinocytosis and phagocytosis by macrophages. J Cell Biol 135(5):1249-1260. https://doi.org/10.1083 /jcb.135.5.1249

Bacon M, Bradley SJ, Nann T (2014) Graphene quantum dots. Part Part Syst Charact 31(4):415-428. https://doi. org/10.1002/ppsc.201300252

Bharti C, Nagaich U, Pal AK, Gulati N (2015) Mesoporous silica nanoparticles in target drug delivery system: a review. Int $\mathrm{J}$ Pharma Investig 5(3):124-133 http://www.jpionline.org/text. asp?2015/5/3/124/160844

Cao M, Wang P, Kou Y, Wang J, Liu J, Li Y, Li J, Wang L, Chen C (2015) Gadolinium (III)-chelated silica nanospheres integrating chemotherapy and photothermal therapy for cancer treatment and magnetic resonance imaging. ACS Appl Mater Inter 7(45):25014-25023. https://doi.org/10.1021/acsami.5 b06938

Chen T, Yu H, Yang N, Wang M, Ding C, Fu J (2014) Graphene quantum dot-capped mesoporous silica nanoparticles through an acid-cleavable acetal bond for intracellular drug delivery and imaging. J Mater Chem B 2(31):4979-4982. https://doi.org/10.1039/C4TB00849A

Deng T, Peng Y, Zhang R, Wang J, Zhang J, Huang GY, Deng D (2017) Water-solubilizing hydrophobic ZnAgInSe/ZnS QDs with tumor-targeted cRGD-sulfobetaine-PIMA-histamine ligands via a self-assembly strategy for bioimaging. ACS Appl Mater Interfaces 9(13):11405-11414. https://doi.org/10.1021 /acsami.6b16639
Ekkapongpisit GA, Follo C, Caputo G, Isidoro C (2012) Biocompatibility, endocytosis, and intracellular trafficking of mesoporous silica and polystyrene nanoparticles in ovarian cancer cells: effects of size and surface charge groups. Int J Nanomedicine 7:4147-4158. https://doi.org/10.2147/IJN. S33803

Frohlich E (2012) The role of surface charge in cellular uptake and cytotoxicity of medical nanoparticles. Int J Nanomedicine 7: 5577-5591. https://doi.org/10.2147/IJN.S36111

Graham L, Orenstein JM (2007) Processing tissue and cells for transmission electron microscopy in diagnostic pathology and research. Nat Protoc 2(10):2439-2450. https://doi. org/10.1038/nprot.2007.304

Hao N, Li L, Zhang Q, Huang X, Meng X, Zhang Y, Chen D, Tang F, Li L (2012) The shape effect of PEGylated mesoporous silica nanoparticles on cellular uptake pathway in Hela cells. Microporous Mesoporous Mater 162:14-23 http://www. sciencedirect.com/science/article/pii/S1387181112003514

Hao YN, Guo HL, Tian L, Kang X (2015) Enhanced photoluminescence of pyrrolic-nitrogen enriched graphene quantum dots. RSC Adv 5(54):43750-43755. https://doi. org/10.1039/C5RA07745A

Huang S, Song L, Xiao Z, Hu Y, Peng M, Li J, Zheng X, Wu B, Yuan C (2016) Graphene quantum dot-decorated mesoporous silica nanoparticles for high aspirin loading capacity and its pH-triggered release. Anal Methods 8(12):2561-2567. https://doi.org/10.1039/C5AY03176A

Kozak O, Sudolska M, Pramanik G, Cigler P, Otyepka M, Zboril R (2016) Photoluminescent carbon nanostructures. Chem Mater 28(12):4085-4128. https://doi.org/10.1021/acs. chemmater.6b01372

Kwolek-Mirek M, Zadrag-Tecza R (2014) Comparison of methods used for assessing the viability and vitality of yeast cells. FEMS Yeast Res 14(7):1068-1079. https://doi. org/10.1111/1567-1364.12202

Lai CY, Trewyn BG, Jeftinija DM, Jeftinija K, Xu S, Jeftinija S, Lin VSY (2003) A mesoporous silica nanosphere-based carrier system with chemically removable CdS nanoparticle caps for stimuli-responsive controlled release of neurotransmitters and drug molecules. J Am Chem Soc 125(15):44514459. https://doi.org/10.1021/ja0286501

Le PU, Guay G, Altschuler Y, Nabi IR (2002) Caveolin-1 is a negative regulator of caveolae-mediated endocytosis to the endoplasmic reticulum. J Biol Chem 277(5):3371-3379 http://www.jbc.org/content/277/5/3371.abstract

Lee JE, Lee N, Kim H, Kim J, Choi SH, Kim JH, Kim T, Song IC, Park SP, Moon WK, Hyeon T (2010) Uniform mesoporous dye-doped silica nanoparticles decorated with multiple magnetite nanocrystals for simultaneous enhanced magnetic resonance imaging, fluorescence imaging, and drug delivery. J Am Chem Soc 132(2):552-557. https://doi.org/10.1021 /ja905793q

Lee JE, Lee N, Kim T, Kim J, Hyeon T (2011) Multifunctional mesoporous silica nanocomposite nanoparticles for theranostic applications. Acc Chem Res 44(10):893-902. https://doi.org/10.1021/ar2000259

Lin YS, Hung Y, Su JK, Lee R, Chang C, Lin ML, Mou CY (2004) Gadolinium (III)-incorporated nanosized mesoporous silica as potential magnetic resonance imaging contrast agents. J Phys Chem B 108(40):15608-15611. https://doi. org/10.1021/jp047829a 
Lu J, Liong M, Sherman S, Xia T, Kovochich M, Nel AE, Zink JI, Tamanoi F (2007) Mesoporous silica nanoparticles for cancer therapy: energy-dependent cellular uptake and delivery of paclitaxel to cancer cells. NanoBiotechnology 3(2):89-95. https://doi.org/10.1007/s12030-008-9003-3

Lu F, Wu SH, Hung Y, Mou CY (2009) Size effect on cell uptake in well-suspended, uniform mesoporous silica nanoparticles. Small 5(12):1408-1413. https://doi.org/10.1002 /smll.200900005

Ma M, Chen H, Chen Y, Wang X, Chen F, Cui X, Shi J (2012) Au capped magnetic core/mesoporous silica shell nanoparticles for combined photothermo-/chemo-therapy and multimodal imaging. Biomaterials 33(3):989-998 http://www. sciencedirect.com/science/article/pii/S0142961211012208

Markovic M, Ristic BZ, Arsikin KM, Klisic DG, Harhaji-Trajkovic LM, Todorovic-Markovic BM, Kepic DP, Kravic-Stevovic TK, Jovanovic SP, Milenkovic MM, Milivojevic DD, Bumbasirevic VZ, Dramicanin MD, Trajkovic VS (2012) Graphene quantum dots as autophagy-inducing photodynamic agents. Biomaterials 33(29):7084-7092 http://www. sciencedirect.com/science/article/pii/S0142961212007107

Meng H, Yang S, Li Z, Xia T, Chen J, Ji Z, Zhang H, Wang X, Lin S, Huang C, Zhou ZH, Zink JI, Nel AE (2011) Aspect ratio determines the quantity of mesoporous silica nanoparticle uptake by a small GTPase-dependent macropinocytosis mechanism. ACS Nano 5(6):4434-4447. https://doi. org/10.1021/nn103344k

Moreira A, Dias DR, Correia IJ (2016) Stimuli-responsive mesoporous silica nanoparticles for cancer therapy: a review. Microporous Mesoporous Mater 236:141-157 http://www. sciencedirect.com/science/article/pii/S1387181116303730

Niu N, He F, Ma PA, Gai S, Yang G, Qu F, Wang Y, Xu J, Yang P (2014) Up-conversion nanoparticle assembled mesoporous silica composites: synthesis, plasmon-enhanced luminescence, and near-infrared light triggered drug release. ACS Appl Mater Interfaces 6(5):3250-3262. https://doi. org/10.1021/am500325w

Nooney RI, Thirunavukkarasu D, Chen Y, Josephs R, Ostafin AE (2002) Synthesis of nanoscale mesoporous silica spheres with controlled particle size. Chem Mater 14:4721-4728 https://pubs.acs.org/doi/abs/10.1021/cm0204371

Parton RG, Richards AA (2003) Lipid rafts and caveolae as portals for endocytosis: new insights and common mechanisms. Traffic 4(11):724-738 https://onlinelibrary.wiley. com/doi/abs/10.1034/j.1600-0854.2003.00128.x

Permatasari FA, Aimon AH, Iskandar F, Ogi T, Okuyama K (2016) Role of C-N configurations in the photoluminescence of graphene quantum dots synthesized by a hydrothermal route. Adv Opt Mater 6:21042. https://doi.org/10.1038 /srep21042

Qu D, Zheng M, Du P, Zhou Y, Zhang L, Li D, Tan H, Zhao Z, Xie Z, Sun Z (2013) Highly luminescent S,N co-doped graphene quantum dots with broad visible absorption bands for visible light photocatalysts. Nanoscale 5(24):12272-12277. https://doi.org/10.1039/C3NR04402E

Qu D, Sun Z, Zheng M, Li J, Zhang Y, Zhang G, Zhao H, Liu X, Xie Z (2014) Three colors emission from S,N Co-doped graphene quantum dots for visible light $\mathrm{H}_{2}$ production and bioimaging. Adv Opt Mater 3(3):360-367. https://doi. org/10.1002/adom.201400549
Rafipoor M, Schmidtke C, Wolter C, Strelow C, Weller H, Lange $\mathrm{H}$ (2015) Clustering of CdSe/CdS quantum dot/quantum rods into micelles can form bright, non-blinking, stable, and biocompatible probes. Langmuir 31(34):9441-9447. https://doi. org/10.1021/acs.langmuir.5b01570

Resch-Genger U, Grabolle M, Cavaliere-Jaricot S, Nitschke R, Nann T (2008) Quantum dots versus organic dyes as fluorescent labels. Nat Methods 5(9):763-775. https://doi. org/10.1038/nmeth. 1248

Rupper A, Lee K, Knecht D, Cardelli J (2001) Sequential activities of phosphoinositide 3-kinase, PKB/Akt, and Rab7 during macropinoso formation in Dictyostelium. Mol Biol Cell 12(9):2813-2824 http://www.molbiolcell.org/content/12 /9/2813.abstract

Santos d, Varela J, Lynch I, Salvati A, Dawson KA (2011) Effects of transport inhibitors on the cellular uptake of carboxylated polystyrene nanoparticles in different cell lines. PLoS One 6(9):e24438. https://doi.org/10.1371/journal.pone.0024438

Schroeder KL, Goreham RV, Nann T (2016) Graphene quantum dots for theranostics and bioimaging. Pharm Res:1-21. https://doi.org/10.1007/s11095-016-1937-x

Sk MA, Ananthanarayanan A, Huang L, Lim KH, Chen P (2014) Revealing the tunable photoluminescence properties of graphene quantum dots. J Mater Chem C 2(34):6954-6960. https://doi.org/10.1039/C4TC01191K

Upreti M, Jyoti A, Sethi P (2013) Tumor microenvironment and nanotherapeutics. Transl Cancer Res 2(4):309-319 http://www.ncbi.nlm.nih.gov/pmc/articles/PMC3951160/

Vollrath A, Schallon A, Pietsch C, Schubert S, Nomoto T, Matsumoto Y, Kataoka K, Schubert US (2013) A toolbox of differently sized and labeled PMMA nanoparticles for cellular uptake investigations. Soft Matter 9(1):99-108. https://doi.org/10.1039/C2SM26928G

Wen J, Xu Y, Li H, Lu A, Sun S (2015) Recent applications of carbon nanomaterials in fluorescence biosensing and bioimaging. Chem Commun 51(57):11346-11358. https://doi.org/10.1039/C5CC02887F

Wu SH, Mou CY, Lin HP (2013a) Synthesis of mesoporous silica nanoparticles. Chem Soc Rev 42(9):3862-3875. https://doi. org/10.1039/C3CS35405A

Wu X, Tian F, Wang W, Chen J, Wu M, Zhao JX (2013b) Fabrication of highly fluorescent graphene quantum dots using L-glutamic acid for in vitro/in vivo imaging and sensing. J Mater Chem C 1(31):4676-4684. https://doi. org/10.1039/C3TC30820K

Yao X, Niu X, Ma K, Huang P, Grothe J, Kaskel S and Zhu YC (2017a) Graphene quantum dots-capped magnetic mesoporous silica nanoparticles as a multifunctional platform for controlled drug delivery, magnetic hyperthermia, and photothermal therapy. Small 13(2): 1602225-n/a. https://doi. org/10.1002/smll.201602225

Yao X, Tian Z, Liu J, Zhu Y, Hanagata N (2017b) Mesoporous silica nanoparticles capped with graphene quantum dots for potential chemo-Photothermal synergistic cancer therapy. Langmuir 33(2):591-599. https://doi.org/10.1021/acs. langmuir.6b04189

Yuan X, Liu Z, Guo Z, Ji Y, Jin M, Wang X (2014) Cellular distribution and cytotoxicity of graphene quantum dots with different functional groups. Nanoscale Res Lett 9(1):1-9. https://doi.org/10.1186/1556-276X-9-108 
Zhang S, Wen L, Yang J, Zeng J, Sun Q, Li Z, Zhao D, Dou S (2016) Facile fabrication of dendritic mesoporous $\mathrm{SiO}_{2} @ \mathrm{CdTe} @ \mathrm{SiO}_{2}$ fluorescent nanoparticles for bioimaging. Part Part Syst Charact 33(5):261-270. https://doi.org/10.1002/ppsc.201500254

Zhu S, Zhang J, Qiao C, Tang S, Li Y, Yuan W, Li B, Tian L, Liu F, $\mathrm{Hu}$ R, Gao H, Wei H, Zhang H, Sun H and Yang B (2011) Strongly green-photoluminescent graphene quantum dots for bioimaging applications. Chem Commun 47. https://doi. org/10.1039/c1cc11122a

Zhu S, Zhang J, Tang S, Qiao C, Wang L, Wang H, Liu X, Li B, Li Y, Yu W, Wang X, Sun H, Yang B (2012) Surface chemistry routes to modulate the photoluminescence of graphene quantum dots: from fluorescence mechanism to up-conversion bioimaging applications. Adv Funct Mater 22(22):47324740. https://doi.org/10.1002/adfm.201201499 